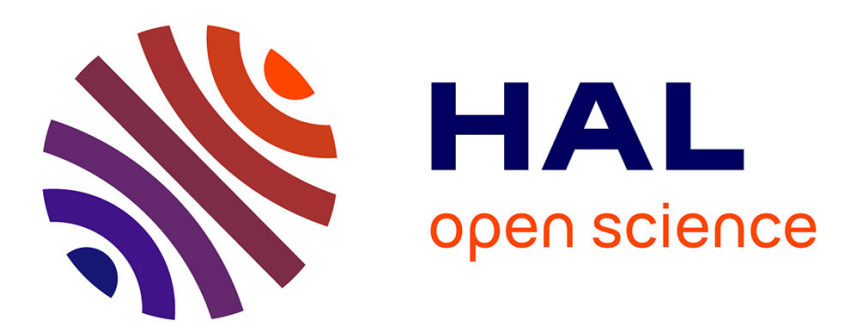

\title{
Construction of continuous functions with prescribed local regularity
}

\author{
Khalid Daoudi, Jacques Lévy Véhel, Yves Meyer
}

\section{To cite this version:}

Khalid Daoudi, Jacques Lévy Véhel, Yves Meyer. Construction of continuous functions with prescribed local regularity. Constructive Approximation, 1998, 14 (3), pp.349-385. 10.1007/s003659900078 . inria-00593268

\section{HAL Id: inria-00593268 \\ https://hal.inria.fr/inria-00593268}

Submitted on 13 May 2011

HAL is a multi-disciplinary open access archive for the deposit and dissemination of scientific research documents, whether they are published or not. The documents may come from teaching and research institutions in France or abroad, or from public or private research centers.
L'archive ouverte pluridisciplinaire HAL, est destinée au dépôt et à la diffusion de documents scientifiques de niveau recherche, publiés ou non, émanant des établissements d'enseignement et de recherche français ou étrangers, des laboratoires publics ou privés. 


\title{
Construction of continuous functions with prescribed local regularity
}

\author{
Khalid DAOUDI†, Jacques LÉVY VÉHEL† and Yves MEYER $\ddagger$ \\ $\dagger$ INRIA Rocquencourt \\ BP 105 - 78153 Le Chesnay cedex FRANCE \\ Tel: (1) 39635473 Fax: (1) 39635330 \\ $\ddagger$ Université Paris IX Dauphine \\ CEREMADE \\ Place du Maréchal de Lattre De Tassigny \\ 75775 Paris FRANCE \\ email: daoudi@bora.inria.fr jlv@bora.inria.fr meyer@ceremade.dauphine.fr
}

\begin{abstract}
In this work we investigate both from a theoretical and a practical point of view the following problem: Let $s$ be a function from $[0 ; 1]$ to $[0 ; 1]$. Under which conditions does there exist a continuous function $f$ from $[0 ; 1]$ to $\mathbb{R}$ such that the regularity of $f$ at $x$, measured in terms of Hölder exponent, is exactly $s(x)$, for all $x \in[0 ; 1]$ ?

We obtain a necessary and sufficient condition on $s$ and give three constructions of the associated function $f$. We also examine some extensions, as for instance conditions on the box or Tricot dimension or the multifractal spectrum of these functions. Finally we present a result on the "size" of the set of functions with prescribed local regularity.
\end{abstract}

\section{Introduction}

Since Riemann [1], a number of authors have been interested in constructing nowhere differentiable continuous functions. Some use geometrical constructions, of which the best known examples are probably Von Koch's [2], Peano's and Hilbert's [3] curves, while others are based on analytical tools. The very well known example is here the Weierstrass function, which was shown by Weierstrass to be continuous and nowhere differentiable [4]. This result was later greatly enhanced by Hardy [5] who showed that

$$
f(x)=\sum_{n=0}^{\infty} b^{n} \cos \left(a^{n} x \pi\right)
$$

has nowhere a finite derivative, provided that

$$
0<b<1, \quad a>1, \quad a b \geq 1 \text {. }
$$

Hardy also analyzed the Hölder conditions satisfied by $f(x)$. If $a b>1$, let $\xi<1$ be defined by $\xi=\frac{\log (1 / b)}{\log a}$. Then, for $h \rightarrow 0$,

$$
|f(x+h)-f(x)|=O\left(|h|^{\xi}\right) \text { for every } x
$$


but

$$
|f(x+h)-f(x)|=o\left(|h|^{\xi}\right) \text { for no } x .
$$

Another example of nowhere differentiable function, which fits well the main ideas of this paper, is the Takagi function [6] defined by:

$$
T(x)=\sum_{j=0}^{\infty} 2^{-j} \theta^{*}\left(2^{j} x\right)
$$

where $\theta^{*}(x)$ is the periodic function of period 1 defined on $[0 ; 1]$ by $\theta^{*}(x)=2 x$ if $0 \leq x \leq 1 / 2$ and $\theta^{*}(x)=2-2 x$ if $1 / 2 \leq x \leq 1$.

Indeed, we consider in the sequal three different constructions of nowhere differentiable functions: one is based on a generalization of the Weierstrass function, another one on an expansion in the Schauder basis, and the last one on a generalization of IFS theory. The construction of the Takagi function bears some analogy with that of the Weierstrass function. On the other hand, the restriction of $T$ to $[0 ; 1]$ can be written, in its expansion in the Schauder basis, as:

$$
T(x)=\sum_{j \geq 0} \sum_{0 \leq k<2^{j}} 2^{-j} \theta\left(2^{j} x-k\right)
$$

where $\theta(x)=\theta^{*}(x)$ if $x \in[0 ; 1]$ and $\theta(x)=0$ if $x \notin[0 ; 1]$. Finally, the graph of the restriction of this function to $[0 ; 1]$ is the attractor of the IFS defined by the two functions $w_{1}(x, y)=\left(\frac{x}{2}, \frac{2 x+y}{2}\right)$ and $w_{2}(x, y)=\left(\frac{x}{2}+\frac{1}{2}, \frac{y-2 x}{2}+1\right)$.

Hata [7] considered the following generalization. Let $g$ be the continuous function defined by:

$$
g(x)=\sum_{n=0}^{\infty} b^{n} q\left(a^{n} x \pi\right)
$$

where $q$ is an almost periodic function and $0<b<1$. He showed in particular that, when $q(x)=\cos (x+\theta)(\theta \in \mathbb{R})$, which leads to :

$$
g(x)=\sum_{n=0}^{\infty} b^{n} \cos \left(a^{n} x \pi+\theta\right),
$$

then the continuous function $g$ has nowhere a finite or infinite derivative if:

$$
a b \geq 1+\pi^{2} .
$$

He also found related results when the function $q$ is almost periodic. This results were later improved by Hu and Lau [8]. Mauldin and Williams [9] also considered a generalization of the Weierstrass function by taking the functions

$$
W_{\beta}(x)=\sum_{-\infty}^{+\infty} \beta^{-\alpha n}\left(\phi\left(\beta^{n} x+\theta_{n}\right)-\phi\left(\theta_{n}\right)\right),
$$

where $\beta>1,0<\alpha<1$, each $\theta_{n}$ is an arbitrary number, and $\phi$ is a function which has period one. They showed that there exists a constant $C>0$ such that, if $\beta$ is large enough, 
then the Hausdorff dimension of the graph of $W_{\beta}$ is bounded from below by $2-\alpha-\frac{C}{\log \beta}$.

Several other techniques are now employed for constructing continuous nowhere differentiable functions. One powerful scheme is to use wavelet decompositions. For instance, Jaffard [10] has given a construction of a function with prescribed multifractal spectrum $(\alpha, f(\alpha))$. Choosing in such a construction $f(\alpha)$ such that $f(\alpha)_{\left.\right|_{]-\infty ; 0] \cup[1 ;+\infty[}}=-\infty$ leads to a nowhere differentiable continuous function.

Another method that has been investigated a lot these past years is based on Iterated Function System (IFS). Although the study of iteration of matrices dates back to Doeblin and Fortet [11] and Dubbins and Freedman [12], it is Hutchinson [13] who really laid the foundations of the IFS theory. Subsequently, several authors have explored this path (see for instance $[14,15,16,17]$ and many others). Barnsley [14] showed that, under some conditions, it is possible to construct an IFS whose attractor is the graph of a continuous nowhere differentiable function. More precise results are now known, concerning the almost sure Hölder exponent of such functions [17] or their multifractal spectrum [18, 19].

We shall hereafter call $\alpha_{f}$ the Hölder function of $f$ (which associates, to each point $x$, the Hölder exponent of the function $f$ at $x$ ).

The main objective of the present work is the following:

Let $s$ be a function from $[0 ; 1]$ to $[0 ; 1]$. Under what conditions on $s$ does there exist a continuous function $f$ from $[0 ; 1]$ to $\mathbb{R}$ such that $\alpha_{f}(x)=s(x)$ for all $x$ in $[0 ; 1]$.

The motivation for this investigation stems partly from applications in signal processing. Indeed, in some cases, it is desirable to model highly irregular signals while precisely controlling the irregularity at each point. This happens, for instance, when the significant information lies in the singularities of the signal more than in its intensity. In such cases, we want to tune the value of $\alpha_{f}(x)$ everywhere and not merely almost everywhere. An example in speech modeling is presented in $[19,20]$.

Our main result is the following:

\section{Theorem}

Let $s$ be a function from $[0 ; 1]$ to $[0 ; 1]$. Then, the following conditions are equivalent:

i) $s$ is the Hölder function of a continuous function $f$ from $[0 ; 1]$ to $\mathbb{R}$.

ii) There exists a sequence $\left(s_{n}\right)_{n \geq 1}$ of continuous functions such that:

$$
s(x)=\liminf _{n \rightarrow+\infty} s_{n}(x), \quad \forall x \in[0 ; 1] .
$$

The proof of i) $\Rightarrow$ ii) is easy and is given in section 3 . The proof of ii) $\Rightarrow$ i) requires more work.

For practical purposes, we are here interested in constructive proofs, i.e, we want to derive explicit methods to construct the function $f$. We present below three such proofs which highlight different aspects of the problem. We also investigate related problems, as for in- 
stance the evaluation of the local box dimension of $f$ at each point or the computation of the multifractal spectrum of $f$. Finally, for practical applications, we want to construct functions $f$ with prescribed Hölder function that satisfies additional constraints, as for instance interpolating a finite number of points $\left(x_{i}, y_{i}\right) \in[0 ; 1] \times \mathbb{R}, i=1,2, \ldots, N$. This naturally leads to a characterization of the set of functions with prescribed Hölder function.

The remainder of this paper is organized as follows: in section 2, we recall some basic definitions about the local regularity of functions, the Hausdorff, Tricot and box dimension. We also prove a new relation between the local box dimension and the Hölder exponent. In section 4 we construct functions with prescribed local regularity $s(x)$ at each point using the Schauder basis. In section 5, we give another solution based on a generalized Weierstrass function. In section 6 , we use IFS to give a solution which constructively allows to interpolate a given finite set of equispaced points. In section 7, we propose some desirable extensions that would allow to measure more finely the local structure of graphs of continuous functions. Section 8 shows some implementation results.

\section{Recalls and a result relating the local box dimen- sion and the Hölder exponent}

In this section we recall some basic definitions useful for the sequel. The definitions are not given in full generality, but only in the form adapted to our problem.

\subsection{Definition of the Hausdorff dimension}

Let $E$ be a non empty set of $\mathbb{R}^{2}$.

Define:

$$
|E|:=\sup _{x, y}\{|x-y| ; x, y \in E\}
$$

to be the diameter of $E$.

If $E \subset \bigcup_{i \in N} E_{i}$ with $0<\left|E_{i}\right| \leq \delta$ for each $i$, then $\left\{E_{i}\right\}_{i \in N}$ is called a (countable) $\delta$-cover of $E$. For $\delta>0$ and $r \geq 0$, define

$$
\mathcal{H}_{\delta}^{r}(E):=\inf \left\{\sum_{i=1}^{+\infty}\left|E_{i}\right|^{r} /\left\{E_{i}\right\}_{i \in N} \delta \text {-cover of } E\right\}
$$

$\mathcal{H}_{\delta}^{r}(E)$ is a non increasing function of $\delta$, and we note

$$
\mathcal{H}^{r}(E):=\lim _{\delta \rightarrow 0} \mathcal{H}_{\delta}^{r}(E)=\sup _{\delta>0} \mathcal{H}_{\delta}^{r}(E)
$$

the Hausdorff r-dimensional outer measure of $E$.

The Hausdorff dimension of $E$ is the unique value $\operatorname{dim}_{H}(E)$ such that [21]:

$$
\mathcal{H}^{r}(E)= \begin{cases}+\infty & \text { if } r<\operatorname{dim}_{\mathrm{H}}(E) \\ 0 & \text { if } r>\operatorname{dim}_{\mathrm{H}}(E)\end{cases}
$$




\subsection{Definition of the box dimension}

For any $\delta>0$, we consider the set of $\delta$-mesh squares in $\mathbb{R}^{2}$ of the form $[i \delta,(i+1) \delta] \times[j \delta,(j+$ $1) \delta$ ] with $i, j$ integers. For any bounded subset $F$ of $\mathbb{R}^{2}$, we denote by $N_{\delta}(F)$ the number of $\delta$-mesh squares which intersect $F$. The box dimension of $F$ is then defined by [22]:

$$
\operatorname{dim}_{B}(F)=\lim _{\delta \rightarrow 0}\left(\frac{\log N_{\delta}(F)}{-\log \delta}\right)
$$

whenever this limit exists.

When the limit exists, its value is unaffected if we change the definition of $N_{\delta}(F)$ and take any of the following:

1. the smallest number of squares of size $\delta$ that cover $F$;

2. the smallest number of closed balls of diameter $\delta$ that cover $F$;

3. the smallest number of sets of diameter $\delta$ that cover $F$;

4. the largest number of disjoint balls of diameter $\delta$ with centers in $F$.

\subsection{Definition of the Tricot (packing) dimension}

Let $F$ be a non empty set of $\mathbb{R}^{2}$, where $n \geq 1$ is an integer, and:

$$
\mathcal{P}_{\delta}^{r}(F)=\sup \left\{\sum_{i \in \mathbb{N}}\left|B_{i}\right|^{r}\right\}
$$

where $\left\{B_{i}\right\}_{i \in N}$ is a collection of disjoint balls of radii at most $\delta$ whose centers belong to $F$. Consider :

$$
\mathcal{P}_{0}^{r}(F)=\lim _{\delta \rightarrow 0} \mathcal{P}_{\delta}^{r}(F)
$$

this limit exists since $\mathcal{P}_{\delta}^{r}(F)$ decreases with $\delta$.

Define now the $r$-dimensional Tricot measure [23, 22] $\mathcal{P}^{r}$ by:

$$
\mathcal{P}^{r}(F)=\inf \left\{\sum_{i=1}^{\infty} \mathcal{P}_{0}^{r}\left(F_{i}\right): F \subset \bigcup_{i=1}^{\infty} F_{i}\right\},
$$

then, the Tricot (or packing) dimension $\operatorname{dim}_{P}$ is defined as follows:

$$
\operatorname{dim}_{P} F=\sup \left\{r: \mathcal{P}^{r}(F)=+\infty\right\}=\inf \left\{r: \mathcal{P}^{r}(F)=0\right\} .
$$

\subsection{Definition of the Hölder spaces and the Hölder exponent}

Let $I$ be an interval in $\mathbb{R}, f$ a continuous function from $I$ to $\mathbb{R}$, and $\beta \in \overline{\mathbb{R}}_{+}^{*} \backslash \mathbb{N}$.

Definition $1 f$ is said to belong to the global Hölder space $C^{\beta}(I)$ iff there exists a positive constant $c$, such that for every $x_{0} \in I$, there exists a polynomial $P_{x_{0}}$ of degree less than or equal to the integer part of $\beta$, such that:

$$
\left|f(x)-P_{x_{0}}\left(x-x_{0}\right)\right| \leq c\left|x-x_{0}\right|^{\beta} \quad \forall x \in I .
$$


Definition 2 Let $t_{0}$ be in $I$. Then $f$ is said to belong to the pointwise Hölder space $C^{\beta}\left(t_{0}\right)$ iff there exists a polynomial $P$ of degree less than or equal to the integer part of $\beta$, and a positive constant $c$ such that, for every $t$ in the neighborhood of $t_{0}$, we have:

$$
\left|f(t)-P\left(t-t_{0}\right)\right| \leq c\left|t-t_{0}\right|^{\beta} .
$$

Recall that if $\beta \in \mathbb{N}^{*}$, the space $C^{\beta}$ must be replaced by the Zygmund $\beta$-class [24].

Definition 3 A function $f$ is said to have Hölder exponent $\beta$ at point $t_{0}$ if $f$ :

i) for every real $\gamma<\beta$ :

$$
\lim _{h \rightarrow 0} \frac{\left|f\left(t_{0}+h\right)-P(h)\right|}{|h|^{\gamma}}=0
$$

ii) if $\beta<+\infty$, for every real $\gamma>\beta$ :

$$
\limsup _{h \rightarrow 0} \frac{\left|f\left(t_{0}+h\right)-P(h)\right|}{|h|^{\gamma}}=+\infty
$$

where $P$ is a polynomial whose degree is less than or equal to the integer part of $\beta$. When $\beta<+\infty$, this is equivalent to :

$$
f \in \bigcap_{\epsilon>0} C^{\beta-\epsilon}\left(t_{0}\right) \text { but } f \notin \bigcup_{\epsilon>0} C^{\beta+\epsilon}\left(t_{0}\right) .
$$

It is also equivalent to:

$$
\beta=\sup \left\{\theta>0: f \in C^{\theta}\left(t_{0}\right)\right\} .
$$

Notice that $f \in C^{\beta}(I)$ does not imply that $\beta=\inf _{t \in I} \alpha_{f}(t)$. As an example, consider the continuous function $f$ defined on $\mathbb{R}$ by:

$$
f(t)=\left\{\begin{array}{cc}
|t| \sin \left(\frac{1}{|t|}\right) & \text { if } t \in \mathbb{R}^{*} \\
0 & \text { if } t=0
\end{array}\right.
$$

then, $f \in C^{\frac{1}{2}}(\mathbb{R})$, but $f$ is $C^{\infty}$ at each point, except at 0 where $\alpha_{f}(0)=1$.

\subsection{A relation between the local box dimension and the Hölder exponent}

In this section we propose a new result that links the local box dimension of the graph of a continuous function and the Hölder function of this function.

Let $f$ be a continuous function from $[0 ; 1]$ to $\mathbb{R}$. We suppose that $s(x)=\alpha_{f}(x) \in[0 ; 1]$ for all $x \in[0 ; 1]$. Let $x \in] 0 ; 1[, \epsilon>0$ such that $] x-\epsilon ; x+\epsilon[\subset[0 ; 1]$ and $\delta \in] 0 ; \epsilon[$.

We cover the plane by a $\delta$-mesh, i.e a grid of squares of the form $[i \delta ;(i+1) \delta] \times[j \delta ;(j+1) \delta]$, with $i, j$ integers.

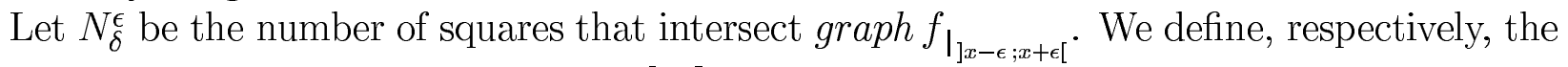
upper and lower local box dimension [22] of the graph of $f$ at the point $x$ by:

$$
\overline{\operatorname{dim}_{B}^{x}} \operatorname{graph} f=\lim _{\epsilon \rightarrow 0} \limsup _{\delta \rightarrow 0}-\frac{\log N_{\delta}^{\epsilon}}{\log \delta}
$$


and

$$
\underline{\operatorname{dim}_{B}^{x}} \operatorname{graph} f=\lim _{\epsilon \rightarrow 0} \liminf _{\delta \rightarrow 0}-\frac{\log N_{\delta}^{\epsilon}}{\log \delta} .
$$

When these numbers coincide, we denote by $\operatorname{dim}_{B}^{x} \operatorname{graph} f$ the local box dimension of $f$ at $x$. For $t \in] 0 ; 1[$ such that $] t-\epsilon ; t+\epsilon[\subset[0,1]$, define:

$$
\bar{c}(t, \epsilon)=\inf \left\{c \in \mathbb{R}_{+}^{*}: \forall \mathrm{u} \in \mathrm{B}(\mathrm{t}, \epsilon),|\mathrm{f}(\mathrm{t})-\mathrm{f}(\mathrm{u})| \leq \mathrm{c}|\mathrm{t}-\mathrm{u}|^{\mathrm{s}(\mathrm{t})}\right\}
$$

and

$$
\underline{c}(t, \epsilon)=\sup \left\{c \in \mathbb{R}_{+}^{*}: \exists \mathrm{u} \in \mathrm{B}(\mathrm{t}, \epsilon):|\mathrm{f}(\mathrm{t})-\mathrm{f}(\mathrm{u})| \geq \mathrm{c} \epsilon^{\mathrm{s}(\mathrm{t})}\right\}
$$

Proposition 1 Let $x$ be a real in $] 0 ; 1[$. Define the following conditions:

there exists $\epsilon^{\prime}>0$ such that $: \bar{C}(x, \epsilon)=\sup _{t \in B(x, \epsilon)} \bar{c}(t, \epsilon)<+\infty$ for every $\epsilon<\epsilon^{\prime} \quad\left(c_{1}\right)$ and

there exists $\epsilon^{\prime}>0$ such that $: \underline{C}(x, \epsilon)=\inf _{t \in B(x, \epsilon)} \underline{c}(t, \epsilon) \neq 0$ for every $\epsilon<\epsilon^{\prime}$

Then, if $\left(c_{1}\right)$ holds, we have the following inequality:

$$
\overline{\operatorname{dim}_{B}^{x}} \operatorname{graph} f \leq 2-\min \left(\liminf _{t \rightarrow x} s(t), s(x)\right)
$$

and, if $\left(c_{2}\right)$ holds, we have:

$$
2-\max \left(\limsup _{t \rightarrow x} s(t), s(x)\right) \leq \underline{\operatorname{dim}_{B}^{x}} \operatorname{graph} f
$$

Proof:

Let $\epsilon$ be a real such that $0<\epsilon<\epsilon^{\prime}$.We denote :

$$
\begin{gathered}
\underline{s}_{x}^{\epsilon}=\inf \{s(t) ; t \in] x-\epsilon ; x+\epsilon[\} . \\
\bar{s}_{x}^{\epsilon}=\sup \{s(t) ; t \in] x-\epsilon ; x+\epsilon[\} . \\
R_{f}\left[t_{1} ; t_{2}\right]=\sup _{t_{1}<u<v<t_{2}}|f(u)-f(v)| .
\end{gathered}
$$

Let $m$ be the least integer greater than or equal to $2 \epsilon / \delta$. Thus, if :

$$
\left.I_{i}(\epsilon, \delta)=\right] x-\epsilon+i \delta ; x-\epsilon+(i+1) \delta[
$$

then:

$$
] x-\epsilon ; x+\epsilon\left[\subset \bigcup_{i=0}^{m-1} I_{i}(\epsilon, \delta) .\right.
$$

However, since $f$ is continuous, the number of squares of the $\delta$-mesh that intersect $g r a p h f_{\left.\right|_{I_{i}(\epsilon, \delta)}}$ is at least $\frac{R_{f}\left(I_{i}(\epsilon, \delta)\right)}{\delta}$ and at most $2+\frac{R_{f}\left(I_{i}(\epsilon, \delta)\right)}{\delta}$. Summing over all such intervals gives :

$$
\delta^{-1} \sum_{i=0}^{m-1} R_{f}\left(I_{i}(\epsilon, \delta)\right) \leq N_{\delta}^{\epsilon} \leq 2 m+\delta^{-1} \sum_{i=0}^{m-1} R_{f}\left(I_{i}(\epsilon, \delta)\right) .
$$


Let now $u, v \in] x-\epsilon ; x+\epsilon[$, with $u<v$. Then:

$$
|f(u)-f(v)| \leq \bar{c}(u, \epsilon)|u-v|^{s(u)},
$$

thus :

$$
|f(u)-f(v)| \leq \bar{C}(x, \epsilon)|u-v|^{\underline{s}_{x}^{\epsilon}} .
$$

We deduce that:

$$
\left.R_{f}\left[t_{1} ; t_{2}\right] \leq \bar{C}(x, \epsilon)\left|t_{1}-t_{2}\right|^{s_{x}^{\epsilon}} \quad \forall t_{1}, t_{2} \in\right] x-\epsilon ; x+\epsilon[,
$$

but $m \leq 1+2 \epsilon \delta^{-1}$, and using (1) we get:

$$
\begin{aligned}
N_{\delta}^{\epsilon} & \leq\left(1+2 \epsilon \delta^{-1}\right)\left(2+\bar{C}(x, \epsilon) \delta^{-1} \delta^{\underline{s}_{x}^{\epsilon}}\right) \\
& \leq c_{1} \epsilon \delta^{\underline{s}_{x}^{\epsilon}-2}
\end{aligned}
$$

where $c_{1}>0$ only depends on $x$ and $\epsilon$ and is finite.

We deduce:

$$
-\frac{\log N_{\delta}^{\epsilon}}{\log \delta} \leq 2-\underline{s}_{x}^{\epsilon}-h(\delta)
$$

where

$$
h(\delta)=\frac{\log c_{1}}{\log \delta}+\frac{\log \epsilon}{\log \delta} .
$$

Since $\lim _{\delta \rightarrow 0} h(\delta)=0$, we obtain :

$$
\limsup _{\delta \rightarrow 0}-\frac{\log N_{\delta}^{\epsilon}}{\log \delta} \leq 2-\underline{s}_{x}^{\epsilon}
$$

which implies:

$$
\lim _{\epsilon \rightarrow 0} \limsup _{\delta \rightarrow 0}-\frac{\log N_{\delta}^{\epsilon}}{\log \delta} \leq 2-\lim _{\epsilon \rightarrow 0} \underline{s}_{x}^{\epsilon}
$$

but

$$
\lim _{\epsilon \rightarrow 0} \underline{s}_{x}^{\epsilon}=\min \left(\liminf _{t \rightarrow x} s(t), s(x)\right)
$$

and finally:

$$
\overline{\operatorname{dim}_{B}^{x}} \operatorname{graph} f \leq 2-\min \left(\liminf _{t \rightarrow x} s(t), s(x)\right) .
$$

Now we establish the other inequality.

For all $v \in] x-\epsilon ; x+\epsilon$, there exists $u$ such that:

$$
|f(u)-f(v)| \geq \underline{c}(v, \epsilon) \epsilon^{s(v)},
$$

thus:

$$
|f(u)-f(v)| \geq \underline{C}(x, \epsilon) \epsilon^{\bar{s}_{x}^{\epsilon}} .
$$

We deduce:

$$
\left.R_{f}\left[t_{1} ; t_{2}\right] \geq \underline{C}(x, \epsilon)\left|t_{1}-t_{2}\right|^{\bar{s}_{x}^{\epsilon}} \quad \forall t_{1}, t_{2} \in\right] x-\epsilon ; x+\epsilon[
$$


but $m \geq 2 \epsilon \delta^{-1}$, and using (1) we get:

$$
\begin{aligned}
N_{\delta}^{\epsilon} & \geq 2 \underline{C}(x, \epsilon) \epsilon \delta^{-1} \delta^{-1} \delta^{\bar{s}_{x}^{\epsilon}} \\
& =2 \underline{C}(x, \epsilon) \epsilon \delta^{\bar{s}_{x}^{\epsilon}-2}
\end{aligned}
$$

Thus:

$$
-\frac{\log N_{\delta}^{\epsilon}}{\log \delta} \geq 2-\bar{s}_{x}^{\epsilon}-h(\delta)
$$

where

$$
h(\delta)=\frac{\log 2 \underline{C}(x, \epsilon) \epsilon}{\log \delta}
$$

Since $\lim _{\delta \rightarrow 0} h(\delta)=0$, we get :

$$
\liminf _{\delta \rightarrow 0}-\frac{\log N_{\delta}^{\epsilon}}{\log \delta} \geq 2-\bar{s}_{x}^{\epsilon}
$$

but

$$
\lim _{\epsilon \rightarrow 0} \bar{s}_{x}^{\epsilon}=\max \left(\limsup _{t \rightarrow x} s(t), s(x)\right)
$$

and finally:

$$
\underline{\operatorname{dim}_{B}^{x}} \operatorname{graph} f \geq 2-\max \left(\limsup _{t \rightarrow x} s(t), s(x)\right) .
$$

This result shows in particular that:

Corollary 1 Whenever $s$ is continuous at point $x$ and conditions $\left(c_{1}\right)$ and $\left(c_{2}\right)$ hold, the local box dimension of $f$ at $x$ exists and is equal to $2-s(x)$.

Note that the converse is not true: the existence of the local box dimension of $f$ at $x$ does not tell anything about the continuity of $s$ at $x$.

Besides, when $s$ is not continuous at $x, s(x)$ and $\operatorname{dim}_{B}^{x}$ graph $f$ can greatly differ (take for instance $f(x)=\sqrt{|x|}$ at $x=0$ ). Another consequence is that we can think of the local box dimension as a more "local" quantity, and of the Hölder exponent as a more "pointwise" quantity: in the case of $f(x)=\sqrt{|x|}$, the local box dimension, which is equal to 1 , is dominated by the local behavior of $f$ around 0 , as the Hölder exponent, $\frac{1}{2}$, reflects the behavior of $f$ solely at 0 .

Let us give an example which shows the necessity of condition $\left(c_{1}\right)$. Consider the continuous function $f$ defined by:

$$
f(x)=\left\{\begin{array}{cl}
x^{u} \cos \left(x^{-v}\right) & \text { if } x \neq 0 \\
0 & \text { if } x=0
\end{array}\right.
$$

where $0<u<v$. This function does not verify condition $\left(c_{1}\right)$. Now, one can prove that, for $x=0, \alpha_{f}(x)=u$ and that $[24, p .126]$ :

$$
\underline{\operatorname{dim}_{B}^{x}} \operatorname{graph} f=\overline{\operatorname{dim}_{B}^{x}} \operatorname{graph} f=2-\frac{u+1}{v+1} .
$$

Hence, when $v<1 / u$, the second inequality in the proposition above does not hold. 


\subsection{A relation between the Tricot dimension and the local Hölder exponent}

Let $f$ be a continuous function on $[0 ; 1]$, and define, for $x \in[0 ; 1]$ and $\epsilon>0$ :

$$
V_{\epsilon}(x)=\sup \left\{\left|f\left(x^{\prime}\right)-f\left(x^{\prime \prime}\right)\right|:\left|x-x^{\prime}\right| \leq \epsilon, \quad\left|x-x^{\prime \prime}\right| \leq \epsilon\right\}
$$

$V_{\epsilon}(x)$ is called the local $\epsilon$-oscillation of $f$ at $x$.

Define now the conditions $\left(p_{1}\right)$ and $\left(p_{2}\right)$ by:

$$
\begin{array}{ll}
\exists s_{1}>0 / \forall x \in[0 ; 1], \exists a_{1}(x)>0 / V_{\epsilon} \leq a_{1}(x) \epsilon^{s_{1}} & \left(p_{1}\right) \\
\exists s_{2}>0 / \forall x \in[0 ; 1], \exists a_{2}(x)>0 / V_{\epsilon} \geq a_{1}(x) \epsilon^{s_{2}} & \left(p_{2}\right) .
\end{array}
$$

Condition $\left(p_{1}\right)$ implies that:

$$
\liminf _{\epsilon \rightarrow 0} \frac{\log V_{\epsilon}(x)}{\log \epsilon} \geq s_{1}
$$

which means that $\alpha_{f}(x) \geq s_{1}$, for every $x \in[0 ; 1]$.

In the same way, condition $\left(p_{2}\right)$ implies that:

$$
\liminf _{\epsilon \rightarrow 0} \frac{\log V_{\epsilon}(x)}{\log \epsilon} \leq s_{2}
$$

which means that $\alpha_{f}(x) \leq s_{2}$, for every $x \in[0 ; 1]$.

Then we have the following result, due to Claude Tricot:

Proposition 2 If condition $\left(p_{1}\right)$ holds, then:

$$
\operatorname{dim}_{P} \text { graph } f \leq \max \left(1,2-s_{1}\right) .
$$

This result remains true when condition $\left(p_{1}\right)$ holds for every $x \in[0 ; 1]$ except on a set $E$ such that $\operatorname{dim}_{P}(E)=0$.

If condition $\left(p_{2}\right)$ holds, then:

$$
\operatorname{dim}_{P} \operatorname{graph} f \geq 2-s_{2} .
$$

This result remains true when condition $\left(p_{2}\right)$ holds for every $x \in[0 ; 1]$ except on a set of Lebesgue measure zero.

\section{Characterization of the set of Hölder functions of continuous function}

Theorem 1 Let $f$ be a nowhere differentiable continuous function from $[0 ; 1]$ to $\mathbb{R}$. Then, there exists a sequence $\left\{s_{n}\right\}_{n \in \mathbb{N}}$ of continuous functions, such that:

$$
\alpha_{f}(x)=\liminf _{n \rightarrow \infty} s_{n}(x) \quad \forall x \in[0 ; 1] .
$$

Conversely, let $s$ be a function from $[0 ; 1]$ to $[0 ; 1]$ such that $s(x)=\liminf _{n \rightarrow \infty} s_{n}(x)$, where the $s_{n}$ 's are continuous functions. Then, there exists a continuous function $f$ from $[0 ; 1]$ to $\mathbb{R}$ such that:

$$
\alpha_{f}(x)=s(x) .
$$


The first part of the theorem is easy to prove. Indeed, take

$$
s_{n}(x)=\inf _{2^{-n} \leq|h|<2^{-n+1}}\left\{\frac{\log \left(|f(x+h)-f(x)|+2^{-n^{2}}\right)}{\log |h|}\right\} .
$$

Then, $s_{n}$ is continuous for every integer $n \geq 1$, and since

$$
\alpha_{f}(x)=\liminf _{h \rightarrow 0} \frac{\log |f(x+h)-f(x)|}{\log |h|},
$$

it is easy to see that:

$$
\alpha_{f}(x)=\liminf _{n \rightarrow \infty} s_{n}(x) \quad \forall x \in[0 ; 1] .
$$

In the following sections, we shall give three constructive proofs of the second part of the theorem. We shall denote by $\mathcal{H}$ the set of all functions, defined from $[0 ; 1]$ to $[0 ; 1]$, which are the lower limit of a sequence of continuous functions.

\section{Construction using the Schauder basis}

This construction is due to S. Jaffard [25], and is based on the well known relation between the pointwise regularity of a function and the coefficients of its expansion in the Schauder basis.

\subsection{Recalls on the Schauder basis}

Consider the function $\theta(x)$ from $\mathbb{R}$ to $\mathbb{R}$ defined by:

$$
\theta(x)=\left\{\begin{array}{cc}
1-|2 x-1| & \text { if } x \in[0 ; 1] \\
0 & \text { if } x \notin[0 ; 1]
\end{array}\right.
$$

It is well known that if $f$ is continuous function from $[0 ; 1]$ to $\mathbb{R}$, and if $f(0)=f(1)=0$, then:

$$
f(x)=\sum_{j \geq 0} \sum_{0 \leq k<2^{j}} c(j, k) \theta_{j, k}(x)
$$

where

$$
\theta_{j, k}(x)=\theta\left(2^{j} x-k\right)
$$

and

$$
c(j, k)=f\left(\left(k+\frac{1}{2}\right) 2^{-j}\right)-\frac{1}{2}\left(f\left(k 2^{-j}\right)+f\left((k+1) 2^{-j}\right)\right) .
$$

We have the following results:

Proposition 3 If $f \in C^{s}\left(x_{0}\right)$, for some $x_{0} \in[0 ; 1]$ and $s>0$, then there exists a constant $C$ such that:

$$
|c(j, k)| \leq C\left(2^{-j}+\left|k 2^{-j}-x_{0}\right|\right)^{s} .
$$

The proof of this proposition is straightforward. 
Proposition 4 Suppose that there exists a constant $C$ such that, for every $x \in[0 ; 1]$ we have:

$$
|f(x+h)-f(x)| \leq C \omega(h) \text { when } h \rightarrow 0
$$

where $\omega$ is a strictly increasing function from $[0 ; 1]$ to $\mathbb{R}$, which verifies:

$$
w(0)=0 \text { and } w(h)=O\left(|\log h|^{-N}\right) \quad \forall N \geq 1 .
$$

Suppose also that, for some $x_{0} \in[0 ; 1]$ and $s>0$, there exists a constant $C$ such that:

$$
|c(j, k)| \leq C\left(2^{-j}+\left|k 2^{-j}+\right| 2^{-j}-x_{0} \mid\right)^{s} .
$$

Then:

$$
f \in C^{s-\epsilon}\left(x_{0}\right) \quad \forall \epsilon>0 .
$$

Proof:

Let $x_{1}$ be a real in the neighborhood of $x_{0}$, and $j_{0}$ be the integer such that

$$
2^{-j_{0}} \leq\left|x_{1}-x_{0}\right|<2^{-\left(j_{0}-1\right)} .
$$

We define the integer $j_{1}$ such that $w\left(2^{-j_{1}}\right)=2^{-s j_{0}}$. Then

$$
\left|f\left(x_{1}\right)-f\left(x_{0}\right)\right| \leq W+X+Y+Z
$$

where

$$
\begin{gathered}
W=\sum_{0 \leq j \leq j_{0}} \sum_{0 \leq k<2^{j}}\left|c(j, k)\left(\theta_{j, k}\left(x_{1}\right)-\theta_{j, k}\left(x_{0}\right)\right)\right| \\
X=\sum_{j>j_{0}} \sum_{0 \leq k<2^{j}}|c(j, k)| \theta_{j, k}\left(x_{0}\right) \\
Y=\sum_{j_{0}<j \leq j_{1}} \sum_{0 \leq k<2^{j}}|c(j, k)| \theta_{j, k}\left(x_{1}\right) \\
Z=\sum_{j>j_{1}} \sum_{0 \leq k<2^{j}}|c(j, k)| \theta_{j, k}\left(x_{1}\right) .
\end{gathered}
$$

For $j<j_{0}-1, \theta_{j, k}\left(x_{0}\right) \neq 0$, implies $\theta_{j, k}\left(x_{1}\right) \neq 0$. Furthermore, for each $j$, there exists a unique $k$ such that $\theta_{j, k}\left(x_{0}\right) \neq 0$ or $\theta_{j, k}\left(x_{1}\right) \neq 0$. In this case, we have $\left|k 2^{-j}-x_{0}\right| \leq 2^{-j}$. Finally, remark that $\left|\theta_{j, k}\left(x_{1}\right)-\theta_{j, k}\left(x_{0}\right)\right| \leq 2^{j}\left|x_{1}-x_{0}\right|$. Hence, we have:

$$
W \leq \sum_{0 \leq j \leq j_{0}} 2^{j(1-s)}\left|x_{1}-x_{0}\right| \leq C\left|x_{1}-x_{0}\right|^{s} .
$$

It is easy to prove that $X \leq C 2^{-j_{0} s}$, which leads to:

$$
X \leq C\left|x_{1}-x_{0}\right|^{s} .
$$

When $\theta_{j, k}\left(x_{1}\right) \neq 0$, we have $\left|k 2^{-j}-x_{1}\right| \leq 2^{-j}$, and if $j>j_{0}$, this implies that:

$$
|c(j, k)| \leq C\left|x_{1}-x_{0}\right|^{s}
$$


hence,

$$
Y \leq C\left(j_{1}-j_{0}\right)\left|x_{1}-x_{0}\right|^{s} .
$$

For every integer $N \geq 1$, there exists a constant $C_{N}$ such that $\omega\left(2^{-j_{1}}\right) \leq C_{N} j_{1}{ }^{-N}$. Hence,

$$
j_{1} \leq C_{N} \frac{1}{N} 2^{j_{0} \frac{s}{N}}
$$

since $\omega\left(2^{-j_{1}}\right)=2^{-j_{0} s}$. This implies that, for every $\epsilon>0$, there exists a constant $C_{\epsilon}$ such that:

$$
j_{1}-j_{0} \leq C_{\epsilon}\left|x_{1}-x_{0}\right|^{-\epsilon} .
$$

Finding an upper bound for $Z$ requires the following results.

Lemma 1 Denote:

$$
S_{q}(f)(x)=\sum_{0 \leq j \leq q} \sum_{0 \leq k<2^{j}} c(j, k) \theta_{j, k}(x),
$$

then $S_{q}(f)$ is the continuous piecewise affine function which satisfies:

$$
S_{q}(f)\left(k 2^{-q}\right)=f\left(k 2^{-q}\right) \quad \forall k=0, \ldots, 2^{q} .
$$

\section{Corollary 2}

$$
\left\|f-S_{q}(f)\right\|_{\infty} \leq \omega\left(2^{-q}\right)
$$

The proofs of the lemma and the corollary are easy.

We remark that $Z \leq\left\|f-S_{j_{1}}(f)\right\|_{\infty}$, and since:

$$
\omega\left(2^{-j_{1}}\right)=2^{-j_{0} s} \leq\left|x_{1}-x_{0}\right|^{s},
$$

the proof of the proposition is completed.

\subsection{Construction of the desired function}

The following result will be used in the proof of the theorem.

Lemma 2 Let $s \in \mathcal{H}$. Then there exists a sequence $\left\{Q_{n}\right\}_{n \geq 1}$ of polynomials such that:

$$
\left\{\begin{array}{l}
s(t)=\liminf _{n \rightarrow+\infty} Q_{n}(t) \quad \forall t \in[0 ; 1] \\
\left\|Q_{n}^{\prime}\right\|_{\infty} \leq n \quad \forall n \geq 1
\end{array}\right.
$$

where $Q_{n}^{\prime}$ is the derivative of $Q_{n}$.

Proof:

Since $s \in \mathcal{H}^{*}$, there exists a sequence $\left\{s_{k}\right\}_{k \in \mathbb{N}^{*}}$ of continuous functions, such that:

$$
s(t)=\liminf _{k \rightarrow+\infty} s_{k}(t) \quad \forall t \in[0 ; 1] .
$$


Thus, there exists a sequence $\left\{P_{k}\right\}$ of polynomials, such that:

$$
s(t)=\liminf _{k \rightarrow+\infty} P_{k}(t) \quad \forall t \in[0 ; 1] .
$$

Let $\left\{q_{k}\right\}_{k \in \mathbb{N}^{*}}$ be a sequence of integers such that:

$$
q_{1} \geq M_{1}
$$

and

$$
q_{k} \geq \max \left(M_{k}, q_{k-1}\right) \text { for } k \geq 2
$$

where

$$
M_{k}=\left\|P_{k}^{\prime}\right\|_{\infty}
$$

Define the sequence $\left\{Q_{j}\right\}_{j \geq 1}$ by:

$$
Q_{j}(t)=0 \text { if } 1 \leq j<q_{1}
$$

and

$$
Q_{j}(t)=P_{k}(t) \text { if } q_{k} \leq j<q_{k+1} \text { for } k \geq 1 .
$$

Of course, $s(t)=\liminf _{j \rightarrow+\infty} Q_{j}(t) \quad \forall t \in[0 ; 1]$. On the other hand

$$
\left|Q_{j}^{\prime}(t)\right|=\left|P_{k}^{\prime}(t)\right| \text { if } q_{k} \leq j<q_{k+1}
$$

and

$$
\left|P_{k}{ }^{\prime}(t)\right| \leq M_{k} \leq q_{k} \quad \forall t \in[0 ; 1]
$$

hence

$$
\left|Q_{j}{ }^{\prime}(t)\right| \leq j \quad \forall j \geq 1 \text { and } \forall t \in[0 ; 1] \square
$$

Proposition 5 Let $s \in \mathcal{H}$ and let $\left(Q_{n}\right)_{n \geq 1}$ be the associated sequence of polynomials verifying (3). Consider the continuous function $f$ defined on $[0 ; 1]$ by:

$$
f(x)=\sum_{j \geq 0} \sum_{0 \leq k<2^{j}} c(j, k) \theta_{j, k}(x)
$$

where

$$
c(k, j)=\inf \left(2^{-\frac{j}{\log j}}, 2^{-j Q_{j}\left(k 2^{-j}\right)}\right) .
$$

Then:

$$
\alpha_{f}(x)=s(x), \quad \forall x \in[0 ; 1] .
$$

Proof:

We first prove that $\alpha_{f}\left(x_{0}\right) \leq s\left(x_{0}\right)$, for every $x_{0} \in[0 ; 1]$.

Let $j \geq 1$ be an integer, and $k$ be the integer such that $x_{0} \in\left[k 2^{-j} ;(k+1) 2^{-j}\right.$ [. Hence, $\left|Q_{j}\left(k 2^{-j}\right)-Q_{j}\left(x_{0}\right)\right| \leq j 2^{-j}$. This implies that, for every $\epsilon>0$ there exists an integer $j_{0}$, such that $c(k, j)>2^{-j\left(s\left(x_{0}\right)+\epsilon\right)}$, for every $j>j_{0}$. Using proposition 3 , we conclude that $\alpha_{f}\left(x_{0}\right) \leq s\left(x_{0}\right)$. 
Let us now show that $\alpha_{f}\left(x_{0}\right) \geq s\left(x_{0}\right)-\epsilon$, for every $\epsilon>0$. Remark that there exists $j_{\epsilon}$ such that:

$$
s\left(x_{0}\right)-\epsilon<Q_{j}\left(k 2^{-j}\right)
$$

for every $j \geq j_{\epsilon}$, and $k$ such that $x_{0} \in\left[k_{0} 2^{-j} ;(k+1) 2^{-j}[\right.$. This implies that:

$$
c(j, k) \leq 2^{-j\left(s\left(x_{0}\right)-\epsilon\right)} .
$$

Furthermore, since $c(j, k) \leq 2^{-\frac{j}{\log j}}$, it is easy to see that condition (2) holds. Hence, we conclude using proposition 4 that $\alpha_{f}\left(x_{0}\right) \geq s\left(x_{0}\right)-\epsilon$. $\square$

\section{$5 \quad$ Use of Weierstrass type functions}

In this section, we show that a simple generalization of the Weierstrass function allows to control the regularity at each point. For a related result, see [24, p.282].

We first recall some properties of the Weierstrass function, which is defined by:

$$
W(t)=\sum_{k=1}^{+\infty} \lambda^{-k s} \sin \left(\lambda^{k} t\right)
$$

where $\lambda>1$ and $s \in] 0 ; 1[$.

It is well known [26] that $\alpha_{W}(t)=s$ for all $t$ and that $\operatorname{dim}_{B}$ graph $W=2-s$. However the value of $\operatorname{dim}_{H}$ graph $W$ is not yet known. Of course: $\operatorname{dim}_{H} \operatorname{graph} W \leq \operatorname{dim}_{B}$ graph $W$, and using mass distribution methods depending on estimates for the Lebesgue measure of the set $\{t /(t, W(t)) \in D\}$ where $D$ is a disc, it can be shown [9] that there exists a constant $c>0$ such that $\operatorname{dim}_{H}$ graph $W \geq s-c / \log \lambda$.

As mentioned in the introduction, several authors have considered generalizations of the Weierstrass function, by replacing the sinus with other type of functions. Here we consider another type of generalization.

Proposition 6 Let $s(t)$ be a function from $[0 ; 1]$ to $[a ; b] \subset] 0 ; 1[$, which is the lower limit of a sequence of continuous functions. Let $a^{\prime}$ and $b^{\prime}$ be two reals such that $0<a^{\prime}<a<b<b^{\prime}<1$ and consider the sequence $\mathbb{L}=\left(l_{p}\right)_{p \geq 1}$ defined by:

$$
\left\{\begin{array}{l}
l_{1}=1 \\
l_{p+1}=\left[\frac{1-a^{\prime}}{1-b^{\prime}} l_{p}\right]+1
\end{array}\right.
$$

where [.] denotes the integer part. Then:

- there exists a sequence $\left\{Q_{n}\right\}_{n \geq 1}$ of polynomials such that:

$$
\left\{\begin{array}{l}
s(t)=\liminf _{\substack{n \rightarrow+\infty \\
n \in \mathbb{L}}} Q_{n}(t) \quad \forall t \in[0 ; 1] \\
\left\|Q_{n}^{\prime}\right\|_{\infty} \leq n \quad \forall n \geq 1
\end{array}\right.
$$

where $Q_{n}^{\prime}$ is the derivative of $Q_{n}$. 
- define:

$$
f(t)=\sum_{k \in \mathbb{L}} \lambda^{-k Q_{k}(t)} \sin \left(\lambda^{k} t\right) .
$$

Then, provided that $\lambda$ is an even integer large enough, we have:

$$
\alpha_{f}(t)=s(t), \forall t \in[0 ; 1] .
$$

Proof:

The proof of the first item is similar to that of Lemma 2 ; the only difference is that we now define the sequence $q_{k}$ by:

$$
q_{k} \geq \max \left(M_{k}, \frac{1-a^{\prime}}{1-b^{\prime}} q_{k-1}+1\right), \text { for } k>1 .
$$

Now, we give the proof of the second item. Let $t$ be fixed and let $\epsilon$ be a positive real such that $s(t)+\epsilon<b^{\prime}$ and $s(t)-\epsilon>a^{\prime}$. We begin by proving that $f \in C^{s(t)-\epsilon}(t)$.

There exists an integer $k_{0}$ such that $Q_{k}(t)>s(t)-\epsilon$, for every $k>k_{0}$. Let $h$ be a real such that $0<|h|<\lambda^{-k_{0}}$. Then we have:

$$
\begin{aligned}
|f(t+h)-f(t)| & =\left|\sum_{k \in \mathbb{L}}\left(\lambda^{-k Q_{k}(t+h)} \sin \left(\lambda^{k}(t+h)\right)-\lambda^{-k Q_{k}(t)} \sin \left(\lambda^{k} t\right)\right)\right| \\
& \leq A+A_{k_{0}}^{\prime}+A^{\prime}
\end{aligned}
$$

where

$$
\begin{gathered}
A=\sum_{k=1}^{+\infty}\left|\left(\lambda^{-k Q_{k}(t+h)}-\lambda^{-k Q_{k}(t)}\right) \sin \left(\lambda^{k}(t+h)\right)\right|, \\
A_{k_{0}}^{\prime}=\sum_{k=1}^{k_{0}} \lambda^{-k Q_{k}(t)}\left|\sin \left(\lambda^{k}(t+h)\right)-\sin \left(\lambda^{k} t\right)\right|,
\end{gathered}
$$

and

$$
A^{\prime}=\sum_{k=k_{0}+1}^{+\infty} \lambda^{-k Q_{k}(t)}\left|\sin \left(\lambda^{k}(t+h)\right)-\sin \left(\lambda^{k} t\right)\right| .
$$

Let us give an upper bound for $A$. We have:

$$
A \leq \sum_{k=1}^{+\infty}\left|\lambda^{-k Q_{k}(t+h)}-\lambda^{-k Q_{k}(t)}\right|
$$

but:

$$
\lambda^{-k Q_{k}(t+h)}-\lambda^{-k Q_{k}(t)}=-(\log \lambda) \times\left[Q_{k}(t+h)-Q_{k}(t)\right] \times\left(k \lambda^{-k \tau}\right)
$$

where $\tau \in\left[\min \left(Q_{k}(t), Q_{k}(t+h)\right) ; \max \left(Q_{k}(t), Q_{k}(t+h)\right)\right]$.

Thus:

$$
A \leq(\log \lambda) \sum_{k=1}^{+\infty} k \lambda^{-k \tau}\left|Q_{k}(t+h)-Q_{k}(t)\right| .
$$


Since

$$
\left|Q_{k}(t+h)-Q_{k}(t)\right| \leq k|h|,
$$

we have :

$$
|A| \leq c_{1}|h| \leq c_{1}|h|^{s(t)-\epsilon},
$$

with $c_{1}=\log \lambda \sum_{k=1}^{+\infty} k^{2} \lambda^{-k a}$.

Let us now give an upper bound for $A^{\prime}$. For this purpose, we consider the integer $N$ such that:

$$
\lambda^{-(N+1)} \leq|h| \leq \lambda^{-N} .
$$

We have, using the mean value theorem:

$$
A^{\prime} \leq|h| X+2 Y
$$

where

$$
X=\sum_{k=1}^{N} \lambda^{-k(s(t)-\epsilon-1)}
$$

and

$$
Y=\sum_{k=N+1}^{+\infty} \lambda^{-k(s(t)-\epsilon)}
$$

but:

$$
\begin{gathered}
X \leq \frac{1}{1-\lambda^{s(t)-1}}|h|^{s(t)-\epsilon-1} \\
Y \leq \frac{1}{1-\lambda^{-s(t)}}|h|^{s(t)-\epsilon} .
\end{gathered}
$$

Since $s(t)$ is bounded, there exists a constant $c_{2}>0$ depending only on $t$ and $\epsilon$ such that:

$$
A^{\prime} \leq c_{2}|h|^{s(t)-\epsilon} .
$$

Finally, it easy to see that there exists a positive constant $c_{3}$, which depends only on $t$ and $\epsilon$, such that:

$$
\left|A_{k_{0}}^{\prime}\right| \leq c_{3}|h| \leq c_{3}|h|^{s(t)-\epsilon} .
$$

Hence, if $c=3 \max \left(c_{1}, c_{2}, c_{3}\right)$, we have:

$$
|f(t+h)-f(t)| \leq c|h|^{s(t)-\epsilon}
$$

Now we will prove that $\alpha_{f}(t) \leq s(t)$.

There exists an infinite set $\Gamma=\Gamma(t, \epsilon) \subset \mathbb{L}$ such that $s(t)-\epsilon<Q_{k}(t)<s(t)+\epsilon$, for every $k \in \Gamma$. Let $N$ be an integer in $\Gamma$ such that $N \gg k_{0}$. Let $h=\frac{\pi}{c(N)} \lambda^{-N}$, where $c(N)$ is chosen in the set $\{ \pm 1, \pm 2\}$ so that:

$$
\left|\sin \left(\lambda^{N} t+\frac{\pi}{c(N)}\right)-\sin \left(\lambda^{N} t\right)\right|>\frac{1}{10} .
$$

Hence, if $\lambda$ is an even integer, we have:

$$
\left|f(t+h)-f(t)-\lambda^{-N Q_{N}(t)}\left(\sin \left(\lambda^{N}(t+h)\right)-\sin \left(\lambda^{N} t\right)\right)\right| \leq A+A_{k_{0}}^{\prime}+A^{\prime \prime}+A^{\prime \prime \prime},
$$


where :

$$
\begin{gathered}
A^{\prime \prime}=\sum_{\substack{k>k_{0} \\
k \in \mathbb{K} \backslash \Gamma}} \lambda^{-k Q_{k}(t)}\left|\sin \left(\lambda^{k}(t+h)\right)-\sin \left(\lambda^{k} t\right)\right| \\
A^{\prime \prime \prime}=\sum_{\substack{k<N \\
k \in \Gamma}} \lambda^{-k Q_{k}(t)}\left|\sin \left(\lambda^{k}(t+h)\right)-\sin \left(\lambda^{k} t\right)\right| .
\end{gathered}
$$

Since $Q_{k}(t) \geq s(t)+\epsilon$ if $k \in \mathbb{L} \backslash \Gamma$ and $k>k_{0}$, we have:

$$
A^{\prime \prime} \leq \sum_{k \in \mathbb{N}} \lambda^{-k(s(t)+\epsilon)}\left|\sin \left(\lambda^{k}(t+h)\right)-\sin \left(\lambda^{k} t\right)\right|
$$

thus, there exists a positive constant $c_{4}$ such that:

$$
A^{\prime \prime} \leq c_{4}|h|^{s(t)+\epsilon} \text {. }
$$

Let $N_{l}$ be the highest integer in $\Gamma$ less than $N$. Then :

$$
\begin{aligned}
A^{\prime \prime \prime} & \leq \sum_{k=0}^{N_{l}} \lambda^{-k(s(t)-\epsilon)}\left|\sin \left(\lambda^{k}(t+h)\right)-\sin \left(\lambda^{k} t\right)\right| \\
& \leq|h| \sum_{k=0}^{N_{l}} \lambda^{k(1-(s(t)-\epsilon))} \\
& \leq|h| \frac{\lambda^{N_{l}(1-(s(t)-\epsilon))}}{\lambda^{1-(s(t)-\epsilon)}-1} .
\end{aligned}
$$

Using the fact that $1-s(t)+\epsilon<1-a^{\prime}$ and $1-s(t)-\epsilon>1-b^{\prime}$, we get:

$$
A^{\prime \prime \prime} \leq|h| \frac{\lambda^{N(1-s(t)-\epsilon)}}{\lambda^{1-(s(t)-\epsilon)}-1} .
$$

Thus, there exists a positive constant $c_{5}$ such that:

$$
A^{\prime \prime \prime} \leq c_{5}|h|^{s(t)+\epsilon} .
$$

We can choose $\lambda$ large enough so that the constants $c_{1}, c_{3}, c_{4}$ and $c_{5}$ are less than $\frac{1}{80}$. Hence we end up with:

$$
|f(t+h)-f(t)|>\frac{1}{20}|h|^{s(t)+\epsilon}
$$

In the case where $s$ is a continous function, we have the following result:

Proposition 7 Let $s$ be a continuous function from $[0 ; 1]$ to $[a ; b] \subset] 0 ; 1[$ such that

$$
s(x)<\alpha_{s}(x) \forall x \in[0 ; 1] .
$$

Assume also that there exsits a constant $M>0$ such that:

$$
|s(t)-s(u)| \leq M|t-u|^{\alpha_{s}(t)} \forall(t, u) \in[0 ; 1] \times[0 ; 1] .
$$

Then, the function $f(x)=\sum_{k \in \mathbb{N}} \lambda^{-k s(x)} \sin \left(\lambda^{k} x\right)$ is such that:

$$
2-\operatorname{dim}_{B}^{x} \operatorname{graph} f=\alpha_{f}(x)=s(x) .
$$

Proof: see appendix. 


\section{Construction using Iterated Function System(IFS)}

The third construction of continuous function with prescribed Hölder function is based upon a generalization of the notion of IFS. This construction bears some analogy with the first one, but here we directly manipulate the contraction ratios of affine functions instead of working on the coefficients of the expansion in the Schauder basis. To begin with, we recall some basic facts about IFS. More details can be found in [13, 27, 16, 17, 15, 28] and others.

\subsection{Recalls}

Let $K$ be a compact metric space whose distance is denoted by $d(x, y)$ for $x, y \in K$. Let $H$ be the set of all non empty closed subsets of $K$. Then $H$ is a compact metric space with the Hausdorff metric [13]:

$$
h(A, B)=\max \left\{\sup _{x \in A} \inf _{y \in B} d(x, y), \sup _{x \in B} \inf _{y \in A} d(x, y)\right\}
$$

which is defined whenever $A$ and $B$ are subsets of $K$.

Let $w_{n}: K \rightarrow K$ for $n \in\{1,2, \ldots, N\}$ be $N$ continuous functions. Then $\left\{K, w_{n}: n=\right.$ $1,2, \ldots, N\}$ is called an iterated function system (IFS). Define $W: H \rightarrow H$ by

$$
W(A)=\bigcup_{n=1}^{N} w_{n}(A) \text { for } A \in H .
$$

Any set $G \in H$ such that

$$
W(G)=G
$$

is called an attractor of the IFS $\left\{K, w_{n}: n=1,2, \ldots, N\right\}$. An IFS always admits at least one attractor. Indeed, start with any $S \in H$, then the closure of the set of all accumulation points of $\left\{W^{o m}(S)\right\}_{m=1}^{\infty}$, with $W^{o m}(S)=W\left(W^{o(m-1)}(S)\right)$, is an attractor of the IFS.

If, for some $s \in[0,1[$ and all $n \in\{1, \ldots, N\}$,

$$
d\left(w_{n}(x), w_{n}(y)\right) \leq s d(x, y), \quad \forall(x, y) \in K \times K
$$

then the IFS is termed hyperbolic. In this case $W$ is a contraction mapping, hence it admits a unique fix point which is the unique attractor of the IFS.

When the attractor $G$ of an IFS is unique, it may be obtained as follows [14]:let $p=$ $\left(p_{1}, \ldots, p_{N}\right)$ be a probability vector with each $p_{n}>0$ and $\sum_{n} p_{n}=1$. Start from the fix point $x_{0}$ of $w_{1}$ and define a sequence $\left(x_{m}\right)$ by choosing successively $x_{m} \in\left\{w_{1}\left(x_{m-1}\right), \ldots, w_{N}\left(x_{m-1}\right)\right\}$ for $m \in\{1,2,3, \ldots\}$, where probability $p_{n}$ is attached to the event $x_{m}=w_{n}\left(x_{m-1}\right)$. Then, the orbit $\left\{x_{m}\right\}_{m \in N}$ is dense in $G$. The $p_{n}$ 's allow to generate a unique probability measure $\mu$ on $K$ which is stationary for the discrete time Markov process defined as follows:

The probability of transfer of $x \in K$ to a Borel subset $B$ of $K$ is :

$$
p(x, B)=\sum_{n} p_{n} \delta_{w_{n}(x)}(B)
$$


where:

$$
\delta_{y}(B)= \begin{cases}1 & \text { if } y \in B \\ 0 & \text { if } y \notin B\end{cases}
$$

We shall not developp here this aspect of IFS theory, and will now focus on the use of IFS for constructing graphs of continuous functions [14].

Given a set of points $\left\{\left(x_{n}, y_{n}\right) \in[0 ; 1] \times[u ; v], n=0,1, \ldots, N\right\}$, with $(u, v) \in \mathbb{R}^{2}$, consider the IFS given by the $N$ contractions $w_{n}(n=1, \ldots, N)$ defined on $[0 ; 1] \times[u ; v]$, by:

$$
w_{n}(x, y)=\left(L_{n}(x) ; F_{n}(x, y)\right)
$$

where $L_{n}$ is a contraction that maps $[0 ; 1]$ to $\left[x_{n-1} ; x_{n}\right]$ and $F_{n}:[0 ; 1] \times[u ; v] \rightarrow[u ; v]$ is a function, contractive with respect to the second variable, such that:

$$
F_{n}\left(x_{0}, y_{0}\right)=y_{n-1} ; F_{n}\left(x_{N}, y_{N}\right)=y_{n}
$$

The attractor of this IFS is the graph of a continuous function $f$ which interpolates the points $\left(x_{n}, y_{n}\right)[14]$.

If the $L_{n}$ 's are affine, $L_{n}(x)=a_{n} x+h_{n}$, and if, for each $n \in\{1, \ldots, N\}$,

$$
t_{n} d(x, y) \leq d\left(w_{n}(x), w_{n}(y)\right) \leq s_{n} d(x, y) \text { for all } x, y \in K
$$

where $0<t_{n} \leq s_{n}<1$, then :

$$
\min (2, l) \leq \operatorname{dim}_{H} \operatorname{graph} f \leq u
$$

where $l$ and $u$ are the positive solutions of:

$$
\sum_{n=1}^{N} t_{n}^{l}=1 \text { and } \sum_{n=1}^{N} s_{n}^{u}=1
$$

and where the lower bound holds when:

$$
t_{1} t_{N} \leq \min \left(a_{1}, a_{N}\right)\left(\sum_{n=1}^{N} t_{n}^{l}\right)^{\frac{2}{l}} .
$$

Concerning the box dimension, if each $F_{n}$ is affine with contraction ratio equal to $c_{n}$, and if the interpolation points are equally spaced, then it is a classical result that [22]:

$$
\operatorname{dim}_{B} \operatorname{graph} f=1+\frac{\log \left(c_{1}+\ldots+c_{N}\right)}{\log N}
$$

\subsection{Local behavior of self-affine functions}

Under some conditions on the $F_{n}$ 's, the function $f$ defined above is nowhere differentiable. But here we want more, namely to control the regularity of $f$ at each point.

In this section we obtain the local Hölder exponent of $f$ at each point $x \in[0 ; 1]$ in the case where the $F_{n}$ 's are affine functions, and the interpolation points are equally spaced . 
We also derive the multifractal spectrum of $f$ and recover the classical formula for the box dimension of the graph of $f$. Related results concerning the almost sure Hölder exponent of $f$ have already been obtained in [17]. Results concerning the multifractal spectrum were independently obtained in [18] and [19].

It is convenient to rewrite our setting in the following form :

Let $S_{i}(0 \leq i<m)$ be affine transformations represented in matrix notation by:

$$
S_{i}\left(\begin{array}{c}
t \\
x
\end{array}\right)=\left(\begin{array}{cc}
1 / m & 0 \\
a_{i} & c_{i}
\end{array}\right)\left(\begin{array}{c}
t \\
x
\end{array}\right)+\left(\begin{array}{c}
i / m \\
b_{i}
\end{array}\right)
$$

We suppose $0 \leq t \leq 1$ and $1 / m<c_{i}<1$. Let $f$ be the function whose graph is the attractor $G$ of the IFS defined by the $S_{i}$ 's (with conditions on $a_{i}$ and $b_{i}$ corresponding to (6) to ensure the continuity of $f$ ). Our result concerning the local regularity of $f$ is the following one:

Proposition 8 Let $0 . i_{1} \ldots i_{k} \ldots$ be the terminating base-m expansion of a real $t \in[0 ; 1)$. Then the Hölder exponent $\alpha$ of $f$ at point $t$ is:

$$
\alpha=\min \left(\liminf _{k \rightarrow+\infty} \frac{\log \left(c_{i_{1}} \ldots c_{i_{k}}\right)}{\log \left(m^{-k}\right)}, \liminf _{k \rightarrow+\infty} \frac{\log \left(c_{j_{1}} \ldots c_{j_{k}}\right)}{\log \left(m^{-k}\right)}, \liminf _{k \rightarrow+\infty} \frac{\log \left(c_{l_{1} \ldots c_{l_{k}}}\right)}{\log \left(m^{-k}\right)}\right)
$$

where, for any positive integer $k$, the $k$-uples $\left(j_{1}, \ldots, j_{k}\right)$ and $\left(l_{1}, \ldots, l_{k}\right)$ of non negative integers strictly smaller than $m$, are uniquely determined by:

$$
\begin{gathered}
t_{k}=m^{-k}\left[m^{k} t\right] \\
\text { if } t_{k}+m^{-k}<1 \text { then } t_{k}^{+}=t_{k}+m^{-k}=\sum_{p=1}^{k} j_{p} m^{-p} \text { else } t_{k}^{+}=t_{k} \\
\text { if } t_{k}-m^{-k}>0 \text { then } t_{k}^{-}=t_{k}-m^{-k}=\sum_{p=1}^{k} l_{p} m^{-p} \text { else } t_{k}^{-}=t_{k}
\end{gathered}
$$

Proof:

The proof is an adaptation of the classical computation of the box dimension of the graph of self affine curves [22].

Let $k$ be a positive integer and $\left(n_{1}, \ldots, n_{k}\right)$ be a $k$-uple of integers such that $0 \leq n_{p}<m$, for every $p=1, \ldots, k$. Let $I_{n_{1} \ldots n_{k}}$ be the interval of reals in $[0 ; 1)$ whose base- $m$ expansion begins with $n_{1} \ldots n_{k}$. Then $g r a p h f_{I_{n_{1} \ldots n_{k}}}=S_{n_{1}} \circ \ldots \circ S_{n_{k}}(G)$, which is a translation of $T_{n_{1}} \circ \ldots \circ T_{n_{k}}(G)$, where $T_{i}$ is the linear part of $S_{i}$. It is easily seen that the matrix representing $T_{n_{1}} \circ \ldots \circ T_{n_{k}}$ is :

$$
\left(\begin{array}{cc}
m^{-k} & 0 \\
m^{1-k} a_{n_{1}}+m^{2-k} c_{n_{1}} a_{n_{2}}+\ldots+c_{n_{1}} c_{n_{2}} \ldots c_{n_{k-1}} a_{n_{k}} & c_{n_{1}} c_{n_{2}} \ldots c_{n_{k}}
\end{array}\right)
$$

Note $a=\max \left|a_{i}\right|, c=\min \left(c_{i}\right), r=\frac{a}{c\left(1-(m c)^{-1}\right)}$. We have:

$$
\left|m^{1-k} a_{n_{1}}+m^{2-k} c_{n_{1}} a_{n_{2}}+\ldots+c_{n_{1}} c_{n_{2}} \ldots c_{n_{k-1}} a_{n_{k}}\right| \leq r c_{n_{1}} \ldots c_{n_{k}},
$$

so that if $s$ is the height of the rectangle containing $G$, then graph $f_{\left.\right|_{I_{n_{1}} \ldots n_{k}}}$ is contained in the rectangle whose height is $(r+s) c_{n_{1}} \ldots c_{n_{k}}$. 
Consider now a real $\beta<\alpha$; there exists a positive integer $k_{0}$ such that, for every integer $k>k_{0}$ we have:

$$
\beta\left(i_{k}\right)>\beta, \quad \beta\left(j_{k}\right)>\beta \text { and } \beta\left(l_{k}\right)>\beta
$$

where

$$
\beta\left(n_{k}\right)=\frac{\log \left(c_{n_{1}} \ldots c_{n_{k}}\right)}{\log \left(m^{-k}\right)} .
$$

Let $h$ be a real small enough so that the integer $k$, defined by $m^{-k-1} \leq|h|<m^{-k}$, verifies $k>k_{0}$. Then either (i), (ii) or (iii) is true:

(i) $(t, t+h) \subset I_{i_{1} \ldots i_{k}}$

(ii) $(t, t+h) \subset I_{i_{1} \ldots i_{k}} \cup I_{j_{1} \ldots j_{k}}$

(iii) $(t, t+h) \subset I_{i_{1} \ldots i_{k}} \cup I_{l_{1} \ldots l_{k}}$.

Denote $r_{1}=r+s$;

case (i): we have :

$$
|f(t+h)-f(t)| \leq r_{1} c_{i_{1}} \ldots c_{i_{k}} .
$$

case (ii): since $f$ is continuous, we have:

$$
|f(t+h)-f(t)| \leq r_{1} c_{i_{1}} \ldots c_{i_{k}}+r_{1} c_{j_{1}} \ldots c_{j_{k}} .
$$

case (iii): using again the continuity of $f$, we have:

$$
|f(t+h)-f(t)| \leq r_{1} c_{i_{1}} \ldots c_{i_{k}}+r_{1} c_{l_{1}} \ldots c_{l_{k}} .
$$

Hence, we always have:

$$
|f(t+h)-f(t)| \leq 2 r_{1}|h|^{\beta} .
$$

This implies that $f \in C^{\alpha-\epsilon}(t)$, for every $\epsilon>0$.

On the other hand, consider now a real $\gamma>\alpha$. Assume w.l.o.g. that:

$$
\alpha=\liminf _{k \rightarrow+\infty} \frac{\log \left(c_{j_{1}} \ldots c_{j_{k}}\right)}{\log \left(m^{-k}\right)},
$$

(the other cases are treated by simply changing $j$ to $i$ or $l$ ).

Then, there exists a subsequence $\sigma(k)$ such that, for every $k$, we have:

$$
\frac{\log \left(c_{j_{1}} \ldots c_{j_{\sigma(k)}}\right)}{\log \left(m^{-\sigma(k)}\right)}<\gamma .
$$

If $q_{1}, q_{2}$ and $q_{3}$ are three non-colinear points in $G$, then $S_{j_{1}} \circ \ldots \circ S_{j_{\sigma(k)}}(G)$ contains the points $\left(x_{n}, f\left(x_{n}\right)\right)=S_{j_{1}} \circ \ldots \circ S_{j_{\sigma(k)}}\left(q_{n}\right)(n=1,2,3)$. The height $d_{\sigma(k)}$ of the triangle with these vertices is at least $d c_{j_{1}} \ldots c_{j_{\sigma(k)}}$ where $d$ is the vertical distance from $q_{2}$ to $\left[q_{1} ; q_{3}\right]$. Thus, for every $k$, there exists a real $h_{k}$ such that $\left|h_{k}\right|<2 m^{-\sigma(k)}$ and

$$
\left|f\left(t+h_{k}\right)-f(t)\right| \geq \frac{d}{2} c_{j_{1}} \ldots c_{j_{\sigma(k)}},
$$

which implies that:

$$
\left|f\left(t+h_{k}\right)-f(t)\right| \geq \frac{d}{2}\left|h_{k}\right|^{\gamma} .
$$


This shows that $f \notin C^{\alpha+\epsilon}(t)$, for every $\epsilon>0$, and the proof is complete.

Using this proposition, it is easy to deduce the spectrum $(\alpha, F(\alpha))$ of singularity of $f$. The proof is analogous to the one for multinomial measures.

Corollary 3 With the same notations as above, and assuming that the proportion $\phi_{i}(t)$ of $(i-1)$ 's in the base-m expansion of $t$ exists for each $i$, we have:

$$
\alpha_{f}(t)=-\sum_{i=0}^{m-1} \phi_{i}(t) \log _{m} c_{i} ; \quad F(\alpha)=-\sum_{i=0}^{m-1} \phi_{i} \log _{m} \phi_{i} ; \tau(q)=-\log _{m} \sum_{i=0}^{m-1} c_{i}^{q}
$$

(for definition of $F$ and $\tau$, see for instance [29].)

Remark 1 Using the relation $\operatorname{dim}_{B}$ graph $f=1-\tau(1)$ we recover the classical result [20]:

$$
\operatorname{dim}_{B} \operatorname{graph} f=1+\log _{m} \sum_{i=0}^{m-1} c_{i}
$$

It is now clear that, with this construction, we can not hope to control the local regularity at each point, since almost all points have the same Hölder exponent (because the almost sure value of $\phi_{i}(t)$ w.r.t the Lebesgue measure is $\left.\frac{1}{m}\right)$. We thus need to use some generalization, which will be presented in the next section.

\subsection{Recursive construction}

We set up here another way to construct fractals recursively, originally due to Anderssson [28]. We consider a collection of sets $\left(F^{k}\right)_{k \in \mathbb{N}^{*}}$, where each $F^{k}$ is a non-empty finite set of contractions $S_{i}^{k}$ in $K$, for $i=0, \ldots, N_{k}-1, N_{k} \geq 1$ being an integer which denotes the cardinal of $F^{k}$. We denote by $c_{i}^{k}$ the contraction ratio of $S_{i}^{k}$, for $i=0, \ldots, N_{k}-1$, and $k \in \mathbb{N}^{*}$.

For $n \in \mathbb{N}^{*}$, let $\Im_{N_{I}}^{n}$ be the set of sequences of length $n$, defined as follows:

$$
\Im_{N_{i}}^{n}=\left\{\sigma=\left(\sigma_{1}, \ldots, \sigma_{n}\right): \sigma_{i} \in\left\{0, \ldots, N_{i}-1\right\}, i \in \mathbb{N}^{*}\right\}
$$

and :

$$
\Im_{N_{i}}^{\infty}=\left\{\sigma=\left(\sigma_{1}, \sigma_{2}, \ldots\right): \sigma_{i} \in\left\{0, \ldots, N_{i}-1\right\}, i \in \mathbb{N}^{*}\right\}
$$

Define the operator $W^{k}: H \rightarrow H$ by:

$$
W^{k}(A)=\bigcup_{n=1}^{N_{k}} S_{n}^{k}(A) \text { for } A \in H
$$

where $N_{k}$ is the cardinal of $F_{k}$. Define the conditions:

$$
\begin{gathered}
\text { (c) } \lim _{n \rightarrow \infty} \sup _{\left(\sigma_{1}, \ldots, \sigma_{n}\right) \in \Im_{N_{i}}^{n}}\left\{\prod_{k=1}^{n} c_{\sigma_{k}}^{k}\right\}=0 \\
\left(c^{\prime}\right) \lim _{n \rightarrow \infty} \sup _{\left(\sigma_{1}, \sigma_{2}, \ldots\right) \in \Im_{N_{i}}^{\infty}}\left\{\sum_{j=n}^{\infty} d\left(S_{\sigma_{j+1}}^{j+1} x, x\right) \prod_{k=1}^{j} c_{\sigma_{k}}^{k}\right\}=0
\end{gathered}
$$

The proof of the following proposition can be found in [28]. 
Proposition 9 If the conditions $(c)$ and $\left(c^{\prime}\right)$ hold, then there exists a unique compact $G$ such that:

$$
\lim _{k \rightarrow \infty} W^{k} \circ \ldots \circ W^{1}(A)=G \text { for every } A \in H .
$$

We call $G$ the attractor of the IFS $\left(K,\left\{F^{k}\right\}_{k \in \mathbb{N}^{*}}\right)$.

We will use this generalized result to obtain more flexibility in the construction of our functions.

Let $F^{k}$ be the set of affine transformations $S_{i}^{k}(0 \leq i<m)$ represented in matrix notation by:

$$
S_{i}^{k}\left(\begin{array}{c}
t \\
x
\end{array}\right)=\left(\begin{array}{cc}
1 / m & 0 \\
a_{i}^{k} & c_{i}^{k}
\end{array}\right)\left(\begin{array}{c}
t \\
x
\end{array}\right)+\left(\begin{array}{c}
i / m \\
b_{i}^{k}
\end{array}\right)
$$

We suppose $0 \leq t \leq 1$ and $1 / m<c_{i}^{k}<1$. We also assume that conditions $(c)$ and $\left(c^{\prime}\right)$ hold to ensure that we have a unique and compact attractor. Then, if the $a_{i}^{k}$ 's and the $b_{i}^{k}$ 's satisfy some relations, analogous to those proposed in subsection 6.1 , one can prove, using the same techniques as in [14], that the attractor of the IFS $\left(K,\left\{F^{k}\right\}_{k \in N}\right)$ is the graph of a continuous function $f$. We, then, have the following result:

Proposition 10 Let $0 . i_{1} \ldots i_{k} \ldots$ be the base-m expansion of a real $t \in[0 ; 1)$. Then:

$$
\alpha_{f}(t)=\min \left(\liminf _{k \rightarrow+\infty} \frac{\log \left(c_{i_{1}}^{1} \ldots c_{i_{k}}^{k}\right)}{\log \left(m^{-k}\right)}, \liminf _{k \rightarrow+\infty} \frac{\log \left(c_{j_{1}}^{1} \ldots c_{j_{k}}^{k}\right)}{\log \left(m^{-k}\right)}, \liminf _{k \rightarrow+\infty} \frac{\log \left(c_{l_{1}}^{1} \ldots c_{l_{k}}^{k}\right)}{\log \left(m^{-k}\right)}\right)
$$

where, for any integer $k$, if we denote $t_{k}=m^{-k}\left[m^{k} t\right]$, the $k$-uples $\left(j_{1}, \ldots, j_{k}\right)$ and $\left(l_{1}, \ldots, l_{k}\right)$ are given by:

$$
\begin{aligned}
& t_{k}^{+}=t_{k}+m^{-k}=\sum_{p=1}^{k} j_{p} m^{-p} \\
& t_{k}^{-}=t_{k}-m^{-k}=\sum_{p=1}^{k} l_{p} m^{-p}
\end{aligned}
$$

Proof:

The proof uses the same techniques as in proposition 8 .

Although this generalization allows more flexibility in the choice of $\alpha_{f}(t)$, it is still too much constrained. Indeed, it is easy to see that if two reals differ only at finite number of ranks in their base- $m$ expansion, then they will have the same Hölder exponent. Hence we can not control the regularity independently at each point.

To do so, let now $F^{k}$ be defined as the set of affine transformations $S_{i}^{k}\left(0 \leq i \leq m^{k}-1\right)$, each $S_{i}^{k}$ operating only on $\left[\left[\frac{i}{m}\right] m^{-k+1} ;\left(\left[\frac{i}{m}\right]+1\right) m^{-k+1}\right]$ and maps to $\left[i m^{-k} ;(i+1) m^{-k}\right]$. Suppose, also, that we want to interpolate the points $\left(\frac{i}{m}, y_{i}\right)$, for $i=0, \ldots, m, m \geq 2$ and $y_{i} \in \mathbb{R}$. Let the compact $K$ be a rectangle containing the $\left(x_{i}, y_{i}\right)$ 's and write:

$$
S_{i}^{k}\left(\begin{array}{c}
t \\
x
\end{array}\right)=\left(\begin{array}{cc}
1 / m & 0 \\
a_{i}^{k} & c_{i}^{k}
\end{array}\right)\left(\begin{array}{c}
t \\
x
\end{array}\right)+\left(\begin{array}{c}
i / m^{k} \\
b_{i}^{k}
\end{array}\right) .
$$


We call $\left(K,\left(F^{k}\right)\right)$ a generalized affine IFS. Define the following conditions, which allow the attractor to be the graph of a continuous function $f$ (for sake of simplicity we will give conditions when $m=2$, the general case being handled similarly): start with the graph of any non affine continuous function $\phi$, and denote:

$$
\phi(0)=u \quad ; \quad \phi(1)=v .
$$

Then choose the contractions (or more precisely the $a_{i}^{k}$ and $b_{i}^{k}$ ) so that they verify the following conditions :

for $i=0,1$ :

$$
\begin{gathered}
S_{i}^{1}(0, u)=\left(\frac{i}{m}, y_{i}\right) ; S_{i}^{1}(1, v)=\left(\frac{i+1}{m}, y_{i+1}\right) \\
S_{0}^{2}\left(0, y_{0}\right)=\left(0, y_{0}\right) ; S_{0}^{2}\left(1 / 2, y_{1}\right)=S_{1}^{2}\left(0, y_{0}\right) ; S_{1}^{2}\left(1 / 2, y_{1}\right)=\left(1 / 2, y_{1}\right) \\
S_{2}^{2}\left(1 / 2, y_{1}\right)=\left(1 / 2, y_{1}\right) ; S_{2}^{2}\left(1, y_{2}\right)=S_{3}^{2}\left(1 / 2, y_{1}\right) ; S_{3}^{2}\left(1, y_{2}\right)=\left(1, y_{2}\right) .
\end{gathered}
$$

for $k>2$ and for $i=0, \ldots, 2^{k}-1$ :

if $i$ is even then:

$$
\begin{gathered}
\text { if } i<2^{k-1}: \\
S_{i}^{k} \circ S_{\frac{i}{2}}^{k-1} \circ S_{\left[\frac{i}{2^{2}}\right]}^{k-2} \circ \ldots \circ S_{\left[\frac{i}{2^{k-2}}\right]}^{2}\left(0, y_{0}\right)=S_{\frac{i}{2}}^{k-1} \circ S_{\left[\frac{i}{2^{2}}\right]}^{k-2} \circ \ldots \circ S_{\left[\frac{i}{2^{k-2}}\right]}^{2}\left(0, y_{0}\right) \\
S_{i}^{k} \circ S_{\frac{i}{2}}^{k-1} \circ S_{\left[\frac{i}{2^{2}}\right]}^{k-2} \circ \ldots \circ S_{\left[\frac{i}{2^{k-2}}\right]}^{2}\left(1 / 2, y_{1}\right)=S_{i+1}^{k} \circ S_{\left[\frac{i+1}{2}\right]}^{k-1} \circ S_{\left[\frac{i+1}{2^{2}}\right]}^{k-2} \circ \ldots \circ S_{\left[\frac{i+1}{2^{k-2}}\right]}^{2}\left(0, y_{0}\right) \\
\text { if } i \geq 2^{k-1}: \\
S_{i}^{k} \circ S_{\frac{i}{2}}^{k-1} \circ S_{\left[\frac{i}{2^{2}}\right]}^{k-2} \circ \ldots \circ S_{\left[\frac{i}{2^{k-2}}\right]}^{2}\left(1 / 2, y_{1}\right)=S_{\frac{i}{2}}^{k-1} \circ S_{\left[\frac{i}{2^{2}}\right]}^{k-2} \circ \ldots \circ S_{\left[\frac{i}{2^{k-2}}\right]}^{2}\left(1 / 2, y_{1}\right) \\
S_{i}^{k} \circ S_{\frac{i}{2}}^{k-1} \circ S_{\left[\frac{i}{2^{2}}\right]}^{k-2} \circ \ldots \circ S_{\left[\frac{i}{2^{k-2}}\right]}^{2}\left(1, y_{2}\right)=S_{i+1}^{k} \circ S_{\left[\frac{i+1}{2}\right]}^{k-1} \circ S_{\left[\frac{i+1}{2^{2}}\right]}^{k-2} \circ \ldots \circ S_{\left[\frac{i+1}{2^{k-2}}\right]}^{2}\left(1 / 2, y_{1}\right)
\end{gathered}
$$

if $i$ is odd, then:

$$
\begin{aligned}
& \text { if } i<2^{k-1}: \\
& S_{i}^{k} \circ S_{\left[\frac{i}{2}\right]}^{k-1} \circ S_{\left[\frac{i}{2^{2}}\right]}^{k-2} \circ \ldots \circ S_{\left[\frac{i}{2^{k-2}}\right]}^{2}\left(1 / 2, y_{1}\right)=S_{\left[\frac{i}{2}\right]}^{k-1} \circ S_{\left[\frac{i}{2^{2}}\right]}^{k-2} \circ \ldots \circ S_{\left[\frac{i}{2^{k-2}}\right]}^{2}\left(1 / 2, y_{1}\right) \\
& \quad \text { if } i \geq 2^{k-1}: \\
& \quad S_{i}^{k} \circ S_{\left[\frac{i}{2}\right]}^{k-1} \circ S_{\left[\frac{i}{2^{2}}\right]}^{k-2} \circ \ldots \circ S_{\left[\frac{i}{2^{k-2}}\right]}^{2}\left(1, y_{2}\right)=S_{\left[\frac{i}{2}\right]}^{k-1} \circ S_{\left[\frac{i}{2^{2}}\right]}^{k-2} \circ \ldots \circ S_{\left[\frac{i}{2^{k-2}}\right]}^{2}\left(1, y_{2}\right) .
\end{aligned}
$$

Our main result is the following: 
Proposition 11 Suppose that conditions $(c)$ and $\left(c^{\prime}\right)$ hold. Then the attractor of the IFS defined above is the graph of a continuous function $f$ such that:

$$
f\left(\frac{i}{m}\right)=y_{i} \forall i=0, \ldots, m
$$

and

$$
\alpha_{f}(t)=\min \left(\alpha_{1}, \alpha_{2}, \alpha_{3}\right)
$$

where

$$
\left\{\begin{array}{l}
\alpha_{1}=\liminf _{k \rightarrow+\infty} \frac{\log \left(c_{m^{k-1} i_{1}+m^{k-2} i_{2}+\ldots+m i_{k-1}+i_{k}}^{k} \ldots c_{m i_{1}+i_{2}}^{2} c_{i_{1}}^{1}\right)}{\log \left(m^{-k}\right)} \\
\alpha_{2}=\liminf _{k \rightarrow+\infty} \frac{\log \left(c_{m^{k-1} j_{1}+m^{k-2} j_{2}+\ldots+m j_{k-1}+j_{k}}^{k} \ldots c_{m j_{1}+j_{2}}^{2} c_{j_{1}}^{1}\right)}{\log \left(m^{-k}\right)} \\
\alpha_{3}=\liminf _{k \rightarrow+\infty} \frac{\log \left(c_{m^{k-1} l_{1}+m^{k-2} l_{2}+\ldots+m l_{k-1}+l_{k}}^{k} \ldots c_{m l_{1}+l_{2}}^{2} c_{l_{1}}^{1}\right)}{\log \left(m^{-k}\right)}
\end{array}\right.
$$

and where the $i_{p}$ 's, $j_{p}$ 's and the $l_{p}$ 's are defined as in proposition 8.

Proof

Let $I_{n_{1} \ldots n_{k}}$ be the interval of reals whose base- $m$ expansion begins with $n_{1} \ldots n_{k}$. Define $G^{k}$ to be the set obtained after $k$ iterations in the process of generation of the attractor $G$, i.e:

$$
G^{k}=W^{k} \circ \ldots \circ W^{1}(G) \text {. }
$$

Then, it easy to see that:

$$
\left.G^{k}\right|_{{ }_{I_{1} \ldots n_{k}}}=S_{m^{k-1} n_{1}+m^{k-2} n_{2}+\ldots+m n_{k-1}+n_{k}}^{k} \circ \ldots \circ S_{m n_{1}+n_{2}}^{2} \circ S_{n_{1}}^{1}(G) .
$$

Using the same techniques as in the proof of proposition 8 , the announced result follows.

Remark 2 Given $m$ reals $\left.r_{1}, \ldots, r_{m} \in\right] \frac{1}{m} ; 1[$, define, for every integer $k \geq 1$ and for every $i \in\left\{0, \ldots, m^{k}-1\right\}$, the $c_{i}^{k}$ 's as follows:

$$
c_{i}^{k}=r_{i+1-m\left[\frac{i}{m}\right]} .
$$

Then, we recover the original construction considered in proposition 8.

The following corollary allows to control the local singularity at each point, while interpolating the points $\left(\frac{i}{m}, y_{i}\right)$, for $i=0, \ldots, m$. We first need to state the following refinement of Lemma 2:

Lemma 3 Let $s \in \mathcal{H}$. Then there exists a sequence $\left\{R_{n}\right\}_{n \geq 1}$ of piecewise polynomials such that:

$$
\left\{\begin{array}{l}
s(t)=\liminf _{n \rightarrow+\infty} R_{n}(t) \quad \forall t \in[0 ; 1] \\
\left\|R_{n}^{\prime+}\right\|_{\infty} \leq n ;\left\|R_{n}^{\prime-}\right\|_{\infty} \leq n \quad \forall n \geq 1 \\
\left\|R_{n}\right\|_{\infty} \geq \frac{1}{\log n}
\end{array}\right.
$$

where $R_{n}^{\prime+}$ and $R_{n}^{\prime-}$ are respectively the right and the left derivative of $R_{n}$. 
Proof:

Let $Q_{k}$ be defined as in Lemma 2 and define:

$$
R_{k}=\max \left(Q_{k}, \frac{1}{\log k}\right)
$$

Corollary 4 Let $s(t)$ be a function from $[0 ; 1]$ to $[0 ; 1]$, which is the lower limit of a sequence of continuous functions.

Then, there exists a generalized affine IFS whose attractor is the graph of a continuous function $f$ which verifies:

$$
\alpha_{f}(t)=s(t)
$$

Proof:

Because of the continuity constraints, finding the generalized affine IFS amounts to determining the double sequence $\left(c_{i}^{k}\right)_{i, k}$.

Let $\left\{R_{n}\right\}_{n \geq 1}$ be a sequence of piecewise polynomials that verifies (8) and let $\mathcal{M}$ be the set of $m$-adic points of $[0 ; 1]$.

Consider now the sequence $\left\{r_{k}\right\}_{k \geq 1}$ of functions from $\mathcal{M}$ to $\mathbb{R}$ defined as follows.

For $t \in \mathcal{M}, t=\sum_{p=1}^{k_{0}} i_{p} m^{-p}$, let:

$$
\begin{gathered}
r_{1}(t)=R_{1}\left(i_{1} m^{-1}\right) \\
r_{k}(t)=k R_{k}(t)-(k-1) R_{k-1}\left(\sum_{p=1}^{k-1} i_{p} m^{-p}\right) \text { for } k=2, \ldots, k_{0}
\end{gathered}
$$

and

$$
r_{k}(t)=k R_{k}(t)-(k-1) R_{k-1}(t) \text { for } k>k_{0} .
$$

Now, for each $k \geq 1$ and $i=0, \ldots, m^{k}-1$, set:

$$
c_{i}^{k}=m^{-r_{k}\left(i m^{-k}\right)} .
$$

Using (9), one verifies that conditions $(c)$ and $\left(c^{\prime}\right)$ are fulfilled.

Using proposition 11, we get:

$$
\alpha_{f}(t)=\min \left(\liminf _{k \rightarrow+\infty} \frac{\sum_{j=1}^{k} r_{j}\left(t_{j}\right)}{k}, \liminf _{k \rightarrow+\infty} \frac{\sum_{j=1}^{k} r_{j}\left(t_{j}^{+}\right)}{k}, \liminf _{k \rightarrow+\infty} \frac{\sum_{j=1}^{k} r_{j}\left(t_{j}^{-}\right)}{k}\right) .
$$

Since :

$$
\begin{gathered}
\frac{\sum_{j=1}^{k} r_{j}\left(t_{j}\right)}{k}=R_{k}\left(t_{k}\right) ; \quad \frac{\sum_{j=1}^{k} r_{j}\left(t_{j}^{+}\right)}{k}=R_{k}\left(t_{k}^{+}\right) ; \frac{\sum_{j=1}^{k} r_{j}\left(t_{j}^{-}\right)}{k}=R_{k}\left(t_{k}^{-}\right), \\
\alpha_{f}(t)=\min \left(\liminf _{k \rightarrow+\infty} R_{k}\left(t_{k}\right), \liminf _{k \rightarrow+\infty} R_{k}\left(t_{k}^{+}\right), \liminf _{k \rightarrow+\infty} R_{k}\left(t_{k}^{-}\right)\right) .
\end{gathered}
$$

Using (8), we have:

$$
\liminf _{k \rightarrow+\infty} R_{k}(t)=\liminf _{k \rightarrow+\infty} R_{k}\left(t_{k}\right)=\liminf _{k \rightarrow+\infty} R_{k}\left(t_{k}^{+}\right)=\liminf _{k \rightarrow+\infty} R_{k}\left(t_{k}^{-}\right) .
$$

We end up with :

$$
\alpha_{f}(t)=s(t)
$$




\section{Concluding remarks}

\subsection{Non uniqueness of $f$}

It is easy to see that, given a set of points $\left\{\left(x_{i}, y_{i}\right)\right\}_{i=0, \ldots, N}$ where $x_{i}=i / N$, and a function $s \in \mathcal{H}$, there is an infinite number of continuous functions that interpolate the $\left(x_{i}, y_{i}\right)$ 's and whose Hölder function is $s$. Indeed, take the function $f$ constructed in section 6.3 and consider the function $g_{\lambda}$ defined by:

$$
g_{\lambda}(x)=\frac{f(x)+\lambda P_{L}(x)}{1+\lambda}
$$

where $P_{L}(x)$ is the Legendre polynomial defined by:

$$
P_{L}(x)=\sum_{i=0}^{N} y_{i} \frac{\prod_{j \neq i}^{N}\left(x-x_{j}\right)}{\prod_{j \neq i}^{N}\left(x_{i}-x_{j}\right)}
$$

and $\lambda$ is a real different from -1 . Then, since $P_{L} \in C^{\infty}(\mathbb{R})$, it is clear that $\alpha_{g_{\lambda}}=s$, and of course, the function $g_{\lambda}$ interpolates the $\left(x_{i}, y_{i}\right)$ 's for every $\lambda \in \mathbb{R} \backslash\{-1\}$.

\subsection{Size of $E_{s}$}

Let $s \in \mathcal{H}$ and define:

$$
E_{s}=\left\{w \in C^{0}([0 ; 1]) / \alpha_{w}(x)=s(x) \forall x \in[0 ; 1]\right\} .
$$

Proposition $12 E_{s}$ is dense in $C^{0}([0 ; 1])$ for the uniform convergence norm $\|\cdot\|_{\infty}$.

Proof:

Let $\mathbb{P}$ be the set of polynomials defined on $[0 ; 1]$. It is well known that $\mathbb{P}$ is dense in $C^{0}([0 ; 1])$ for the uniform convergence norm. For $f \in C^{0}([0 ; 1])$, let $\left(P_{n}\right)_{n \in \mathbb{N}}$ be a sequence such that $P_{n} \in \mathbb{P}$ for every $n \in \mathbb{N}$ and

$$
\left\|P_{n}-f\right\|_{\infty} \rightarrow 0 \text { when } n \rightarrow \infty .
$$

Let now $w$ be a function in $E_{s}$, and consider the sequence $\left(f_{n}\right)_{n \in \mathbb{N}^{*}}$ defined by:

$$
f_{n}=P_{n}+\frac{w}{n} \text { for every } n \in \mathbb{N}^{*} .
$$

Since $P_{n} \in C^{\infty}([0 ; 1])$ for every $n \in \mathbb{N}$, and $w \in E_{s}$, it is clear that $f_{n}$ is in $E_{s}$ for every $n \in \mathbb{N}^{*}$. We have:

$$
\left\|f_{n}-f\right\|_{\infty} \leq\left\|P_{n}-f\right\|_{\infty}+\frac{\|w\|_{\infty}}{n}
$$

$w$ is a continuous function on a compact, and there exists a constant $C>0$ such that $\|w\|_{\infty} \leq C$, hence:

$$
\left\|f_{n}-f\right\|_{\infty} \rightarrow 0 \text { when } n \rightarrow \infty
$$




\subsection{More refined ways of characterizing the local regularity}

The local regularity of the graphs of the functions constructed with the three methods we have presented above appears, in some cases, strikingly different (see section 8). Several improvements may be proposed in order to describe these discrepancies:

- A well known method to measure more precisely the local structure would be to use finer scales of functions, as for instance functions of the form:

$$
g(x)=x^{\alpha}\left(\log \frac{1}{x}\right)^{\beta_{1}}\left(\log \log \frac{1}{x}\right)^{\beta_{2}} \ldots\left(\log \log \ldots \log \frac{1}{x}\right)^{\beta_{n}},
$$

the Hölder exponent at a point $x_{0}$ would then be a vector $\left(\alpha, \beta_{1}, \beta_{2}, \ldots, \beta_{n}\right)$.

- Another possibility is to characterize algebraic oscillations instead of taking the absolute values, i.e consider the two limits:

$$
\limsup _{h \rightarrow 0} \frac{g_{-}(h)}{h^{\gamma}} \text { and } \limsup _{h \rightarrow 0} \frac{g_{+}(h)}{h^{\gamma}},
$$

where

$$
g(x)=f\left(x_{0}+h\right)-f\left(x_{0}\right), g_{+}(x)=\max (g(x), 0), g_{-}(x)=\min (g(x), 0) .
$$

- Finally, especially for practical purposes, the speed of convergence to the local Hölder exponent at $x_{0}$ is of crucial importance. For instance, it is easy to show that, for the Schauder type function considered in section 4 , if we take $s(x)=x$, then, for $x_{0}>0$ and for some sequence $h_{n} \rightarrow 0$, the best possible lower bound is :

$$
\left|f\left(x_{0}+h_{n}\right)-f\left(x_{0}\right)\right| \geq c_{1}\left|h_{n}\right|^{x_{0}-c_{2}\left|h_{n}\right|}
$$

where $c_{1}$ and $c_{2}$ are constants. But for the Weierstrass like functions of section 3 , and also with $s(x)=x$, the best possible lower bound is :

$$
\left|f\left(x_{0}+h_{n}\right)-f\left(x_{0}\right)\right| \geq c^{\prime} h_{n}^{x_{0}}
$$

where $c^{\prime}$ is a constant.

When working with discrete data, this first order difference in $h$ can make a big difference (see figures in the next section).

\section{Examples}

The following figures are graphs of continuous functions with prescribed local regularity. We have implemented the constructions described in section $4,5,6$, and for each case, we show an example with $s(t)=t$ and $s(t)=|\sin (5 \pi t)|$. 
Figure 1: Construction using the Schauder basis with $s(t)=t$

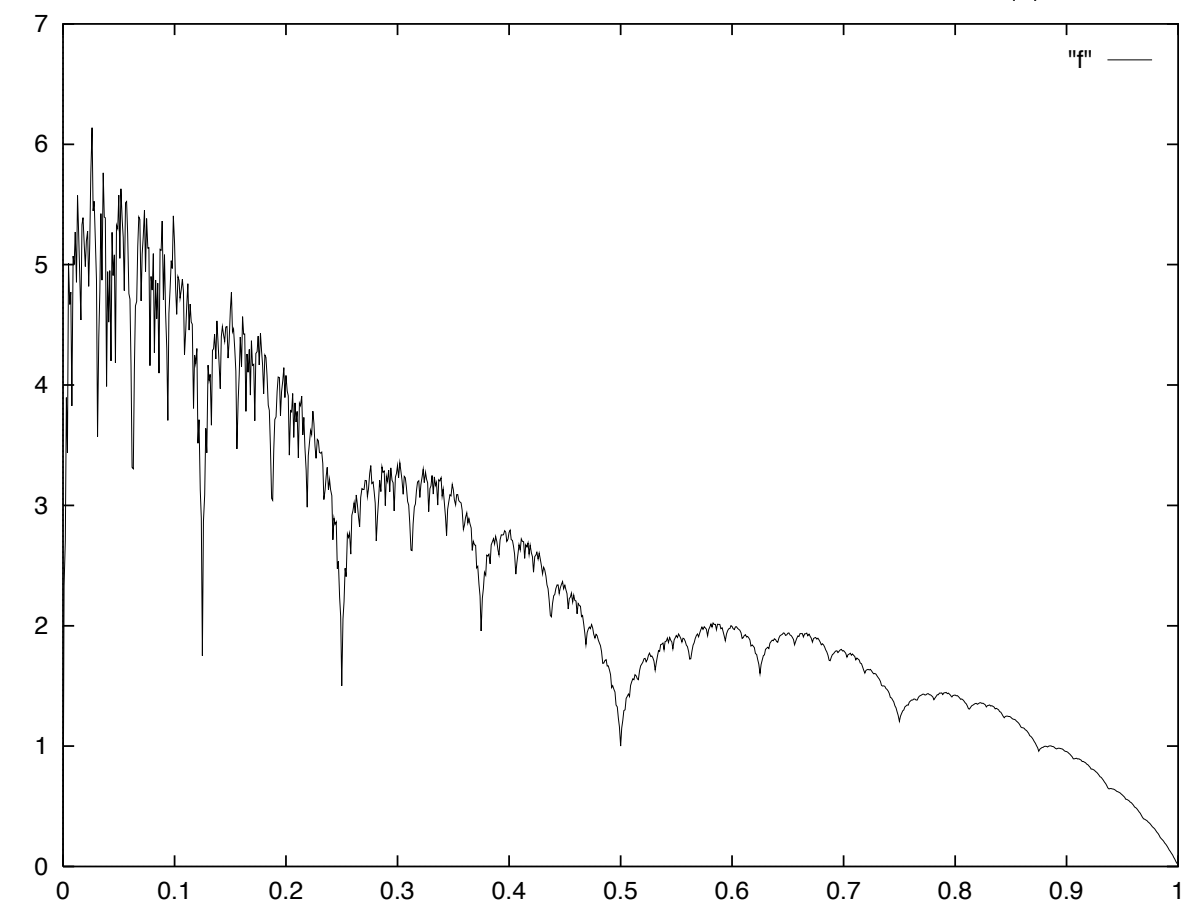

Figure 2: Construction using the Schauder basis with $s(t)=|\sin (5 \pi t)|$

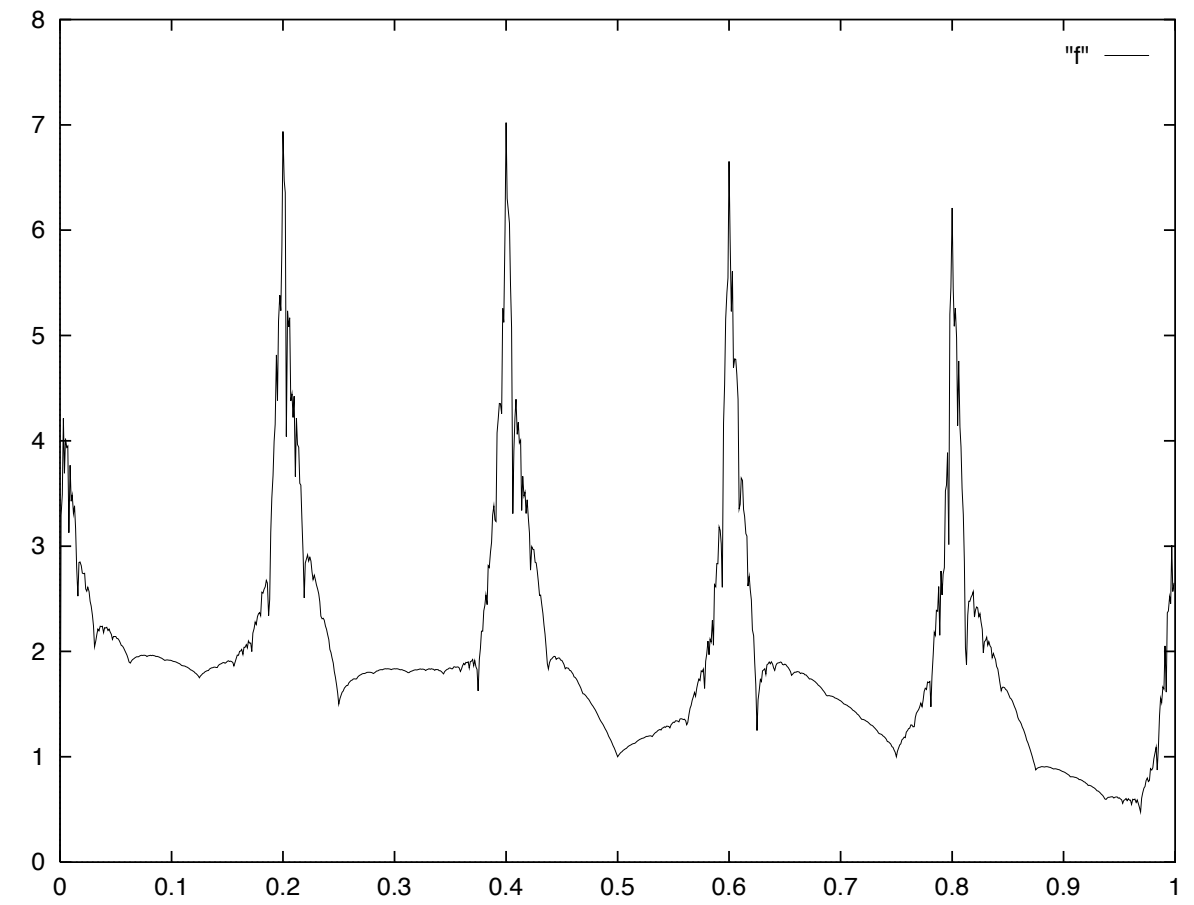


Figure 3: Construction using the Weierstrass type function with $s(t)=t$

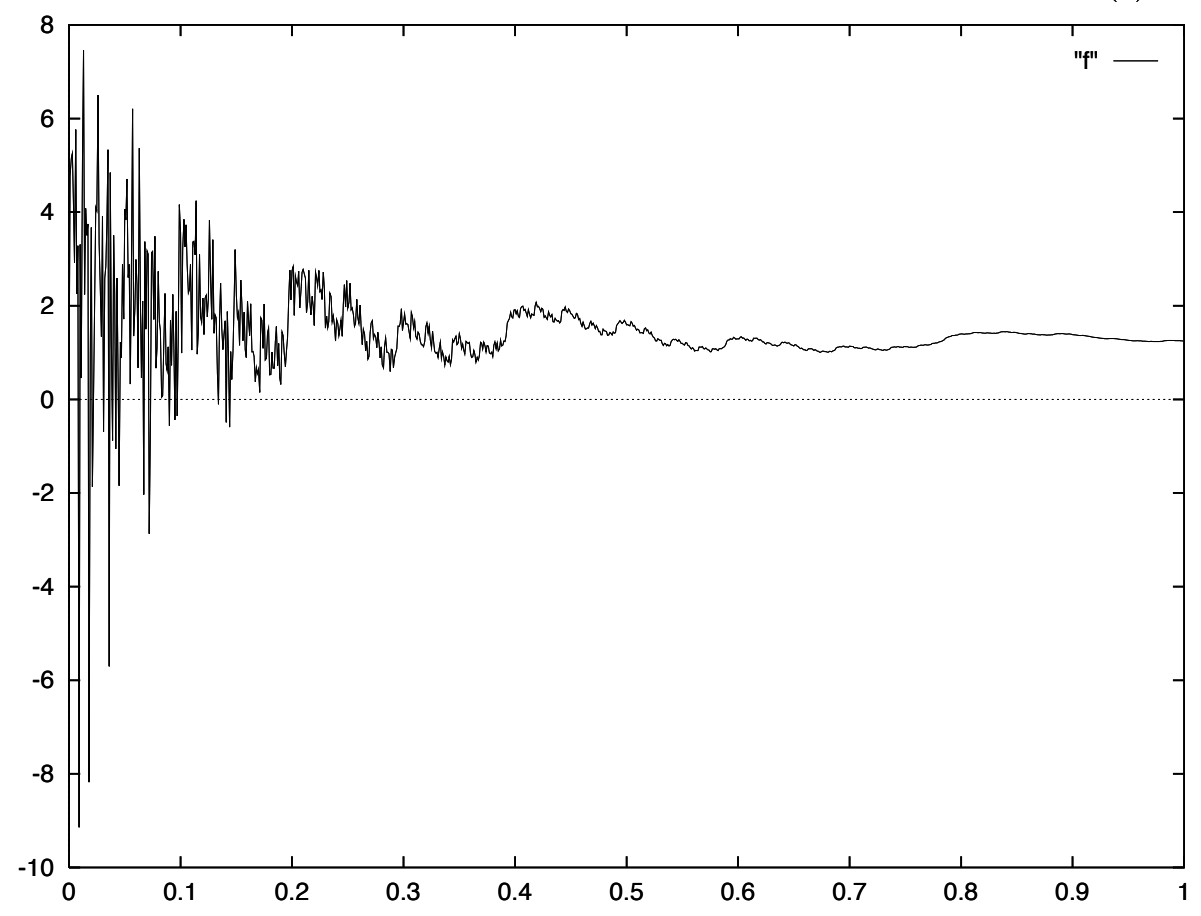

Figure 4: Construction using the Weierstrass type function with $s(t)=|\sin (5 \pi t)|$

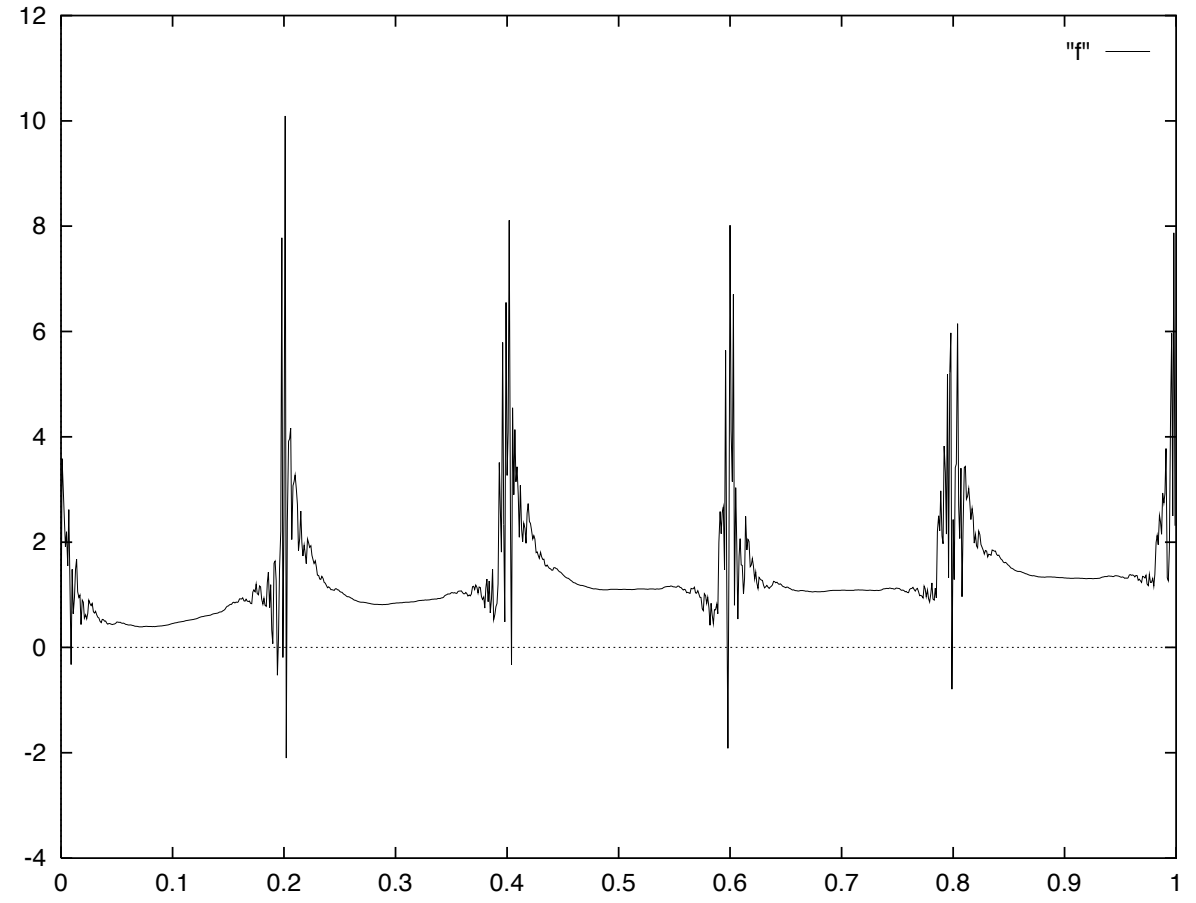


Figure 5: Construction using generalized affine IFS with $s(t)=t$

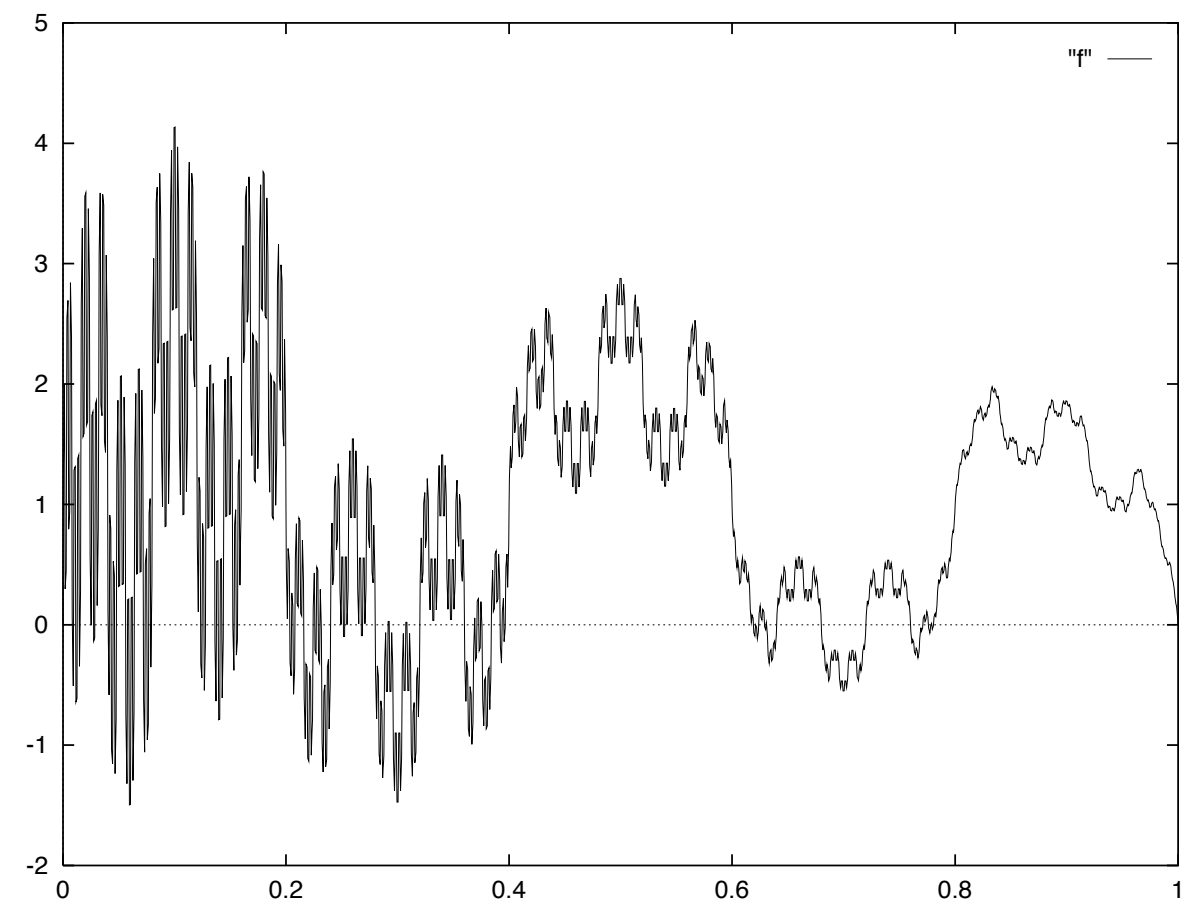

Figure 6: Construction using generalized affine IFS with $s(t)=|\sin (5 \pi t)|$

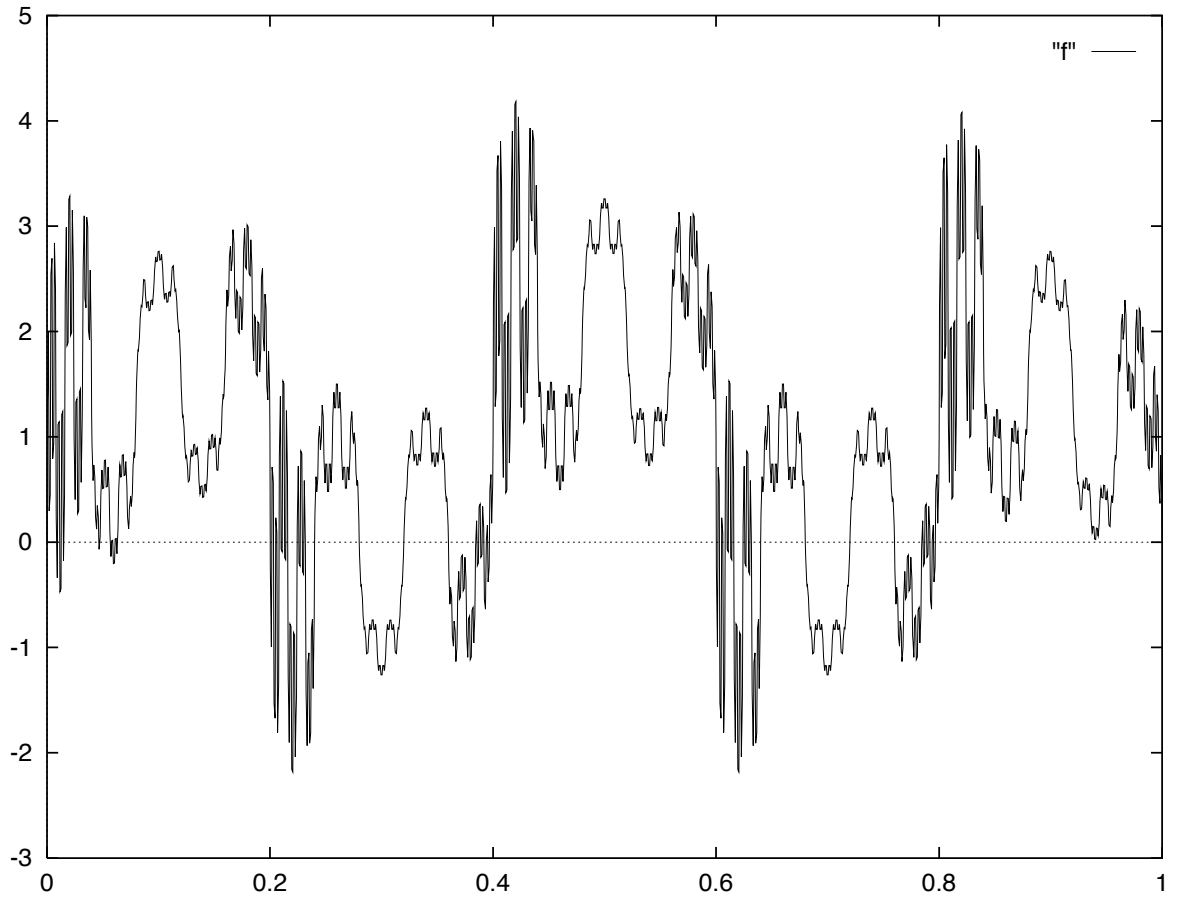




\section{Appendix}

\section{Proof of proposition 7}

Recall that $\alpha_{s}$ is the Hölder function of $s$. We begin by proving that $\alpha_{f}(t) \geq s(t)$.

Let $t$ be fixed, $\epsilon_{2}$ be a real such that $0<\epsilon_{2} \ll 1$, and $h$ be a real such that $0<|h|<\epsilon_{2}$. Then we have:

$$
\begin{aligned}
f(t+h)-f(t) & =\sum_{k=1}^{+\infty}\left(\lambda^{-k s(t+h)} \sin \left(\lambda^{k}(t+h)\right)-\lambda^{-k s(t)} \sin \left(\lambda^{k} t\right)\right) \\
& =A+A^{\prime}
\end{aligned}
$$

where

$$
A=\sum_{k=1}^{+\infty}\left(\lambda^{-k s(t+h)}-\lambda^{-k s(t)}\right) \sin \left(\lambda^{k}(t+h)\right)
$$

and

$$
A^{\prime}=\sum_{k=1}^{+\infty} \lambda^{-k s(t)}\left(\sin \left(\lambda^{k}(t+h)\right)-\sin \left(\lambda^{k} t\right)\right) .
$$

Let us give an upper bound for $|A|$. We have:

$$
|A| \leq \sum_{k=1}^{+\infty}\left|\lambda^{-k s(t+h)}-\lambda^{-k s(t)}\right|
$$

but:

$$
\lambda^{-k s(t+h)}-\lambda^{-k s(t)}=-(\log \lambda) \times[s(t+h)-s(t)] \times\left(k \lambda^{-k \tau}\right)
$$

where $\tau \in[\min (s(t), s(t+h)) ; \max (s(t), s(t+h))]$.

Thus:

$$
|A| \leq(\log \lambda)|s(t+h)-s(t)| \sum_{k=1}^{+\infty} k \lambda^{-k \tau}
$$

Let $C=\sum_{k=1}^{+\infty} k \lambda^{-k \tau}(0<C<+\infty$ because this series converges $)$, then, since there exists a constant $M>0$ such that:

$$
|s(t+h)-s(t)| \leq M|h|^{\alpha_{s}(t)}
$$

we have:

$$
|A| \leq c_{1}|h|^{\alpha_{s}(t)} \leq c_{1}|h|^{s(t)}
$$

where

$$
c_{1}=C M \log \lambda .
$$

Let us now give an upper bound for $\left|A^{\prime}\right|$. For this purpose, we consider the integer $N$ such that:

$$
\lambda^{-(N+1)} \leq|h| \leq \lambda^{-N} \text {. }
$$


We have, using the main value theorem:

$$
\left|A^{\prime}\right| \leq|h| X+2 Y
$$

where

$$
X=\sum_{k=1}^{N} \lambda^{-k(s(t)-1)}
$$

and

$$
Y=\sum_{k=N+1}^{+\infty} \lambda^{-k s(t)}
$$

but:

$$
\begin{gathered}
X \leq \frac{1}{1-\lambda^{s(t)-1}}|h|^{s(t)-1} \\
Y \leq \frac{1}{1-\lambda^{-s(t)}}|h|^{s(t)}
\end{gathered}
$$

Since $s(t)$ is bounded, there exists a constant $c_{2}>0$ such that:

$$
\left|A^{\prime}\right| \leq c_{2}|h|^{s(t)}
$$

Finally, if $c=c_{1}+c_{2}$, we have:

$$
|f(t+h)-f(t)| \leq c|h|^{s(t)}
$$

which gives:

$$
(\gamma<s(t)) \Rightarrow \lim _{h \rightarrow 0} \frac{|f(t+h)-f(t)|}{|h|^{\gamma}}=0 .
$$

Now we will prove that $\alpha_{f}(t) \leq s(t)$.

Let $t$ be a real in $[0 ; 1]$ and $\delta$ a real in $] 0 ; \epsilon_{2}[$. Then, consider the integer $N$ such that $\lambda^{-(N+1)}<\delta \leq \lambda^{-N}$, and let $h$ be a real such that $\lambda^{-(N+1)}<|h| \leq \delta$. We have:

$$
\begin{aligned}
X & =\mid f(t+h)-f(t)-\lambda^{-N s(t)}\left(\sin \left(\lambda^{N}(t+h)-\sin \left(\lambda^{N} t\right)\right) \mid\right. \\
& \leq B+2 \sum_{k=N}^{+\infty} \lambda^{-k s(t)}+|A|
\end{aligned}
$$

where $B=\sum_{k=1}^{N-1} \lambda^{-k s(t)} \mid \sin \left(\lambda^{k}(t+h)-\sin \left(\lambda^{k} t\right) \mid\right.$.

We have:

$$
B \leq \lambda^{-N s(t)} \frac{\lambda^{s(t)-1}}{1-\lambda^{(s(t)-1)}}
$$

Since we have seen that:

$$
|A| \leq c_{1}|h|^{s(t)} \leq c_{1} \lambda^{-N s(t)}
$$

then :

$$
X \leq \lambda^{-N s(t)}\left(c_{1}+c_{3}\right)
$$


with

$$
c_{3}=\frac{\lambda^{s(t)-1}}{1-\lambda^{(s(t)-1)}}+2 \frac{\lambda^{-s(t)}}{1-\lambda^{-s(t)}} .
$$

Provided that $\lambda$ is large enough, we may choose $c_{1}$ and $c_{3}$ such that:

$$
c_{1} \leq \frac{1}{40} \text { and } \quad c_{3} \leq \frac{1}{40}
$$

thus :

$$
X \leq \frac{1}{20} \lambda^{-N s(t)}
$$

but:

$$
X \geq|| f(t+h)-f(t)\left|-\lambda^{-N s(t)}\right| \sin \left(\lambda^{N}(t+h)\right)-\sin \left(\lambda^{N} t\right)||
$$

and :

$$
|f(t+h)-f(t)| \geq \lambda^{-N s(t)}\left|\sin \left(\lambda^{N}(t+h)\right)-\sin \left(\lambda^{N} t\right)\right|-X .
$$

There exists a sequence [22] $\left(h_{n}\right)$, with $\lambda^{-(N+1)}<\left|h_{n}\right| \leq \delta \leq \lambda^{-N}$ for every $n$, such that:

$$
\left|\sin \left(\lambda^{N}\left(t+h_{n}\right)\right)-\sin \left(\lambda^{N} t\right)\right| \geq \frac{1}{10} \forall n
$$

because $\frac{1}{\lambda} \leq\left|h_{n}\right| \lambda^{N} \leq 1 \quad \forall n$

We deduce:

$$
\left|f\left(t+h_{n}\right)-f(t)\right| \geq \frac{1}{20} \lambda^{-N s(t)} \geq \frac{1}{20} \delta^{s(t)} \geq \frac{1}{20}\left|h_{n}\right|^{s(t)}
$$

which gives :

$$
(\gamma>s(t)) \Rightarrow \limsup _{h \rightarrow 0} \frac{|f(t+h)-f(t)|}{|h|^{\gamma}}=+\infty .
$$

Let us now check that $f$ verifies conditions $\left(c_{1}\right)$ and $\left(c_{2}\right)$ of proposition 1 .

Let $x$ be a real in $[0 ; 1]$ and $\epsilon$ be a real such that $0<\epsilon<\min \left(\epsilon_{1}, \epsilon_{2}\right)$. For every $\delta<\epsilon$ and $t \in B(x, \delta)$, we have seen that:

$$
|f(t)-f(u)| \leq\left[c(t) M \log \lambda+\frac{1}{1-\lambda^{s(t)-1}}+\frac{2}{1-\lambda^{-s(t)}}\right]|t-u|^{s(t)} \text { for every } u \in B(t, \delta),
$$

where:

$$
c(t)=\sum_{k=1}^{\infty} k \lambda^{-k \tau} \text { with } \tau \in[\min (s(t), s(u)) ; \max (s(t), s(u))] .
$$

This implies that:

$$
\bar{c}(t, \delta) \leq A M+B \text { for every } t \in[0 ; 1] \text { and } \delta<\epsilon
$$

where:

$$
A=\log \lambda \sum_{k=1}^{\infty} k \lambda^{-k a} \text { and } B=\frac{1}{1-\lambda^{b-1}}+\frac{2}{1-\lambda^{-b}}
$$

Hence :

$$
\bar{C}(x, \delta)<+\infty \quad \forall \delta<\epsilon
$$


and the condition $\left(c_{1}\right)$ holds.

Condition $\left(c_{2}\right)$ is easy to verify. Indeed, we have seen that there exists a real $u \in B(t, \delta)$,such that:

$$
|f(t)-f(u)| \geq \frac{1}{20} \delta^{s(t)}
$$

hence :

$$
\underline{c}(t, \delta) \geq \frac{1}{20} \forall \delta<\epsilon
$$

which implies that:

$$
\underline{C}(x, \epsilon) \neq 0 \text {. }
$$

Now, since $s$ is continuous, and conditions $\left(c_{1}\right)$ and $\left(c_{2}\right)$ hold, we get using proposition 1 :

$$
2-\operatorname{dim}_{B}^{x} \operatorname{graph} f=s(x) \text { for every } x \in[0 ; 1] \text {. }
$$

\section{References}

[1] Gerald A. Edgar, editor. Classics On Fractals. Addison-Wesley Publishing Company, 1993.

[2] Helge von Koch. On a Continuous Curve Tangent Constructible from Elementary Geometry. Arkiv för Matematik, Astronomi och Fysik, pages 681-702, 1904.

[3] E.W. Hobson. The Theory of Functions of Real Variable and the Theory of Fourier's Series. Cambridge University Press, third edition, 1950.

[4] Karl Weierstrass. On Continuous Function of a Real Argument that do not have a Well-Defined Differential Quotient. Mathematische Werke, pages 71-74, 1895.

[5] G.H. Hardy. On Weierstrass's Non-Differentiable Function. Trans. Am. Math. Soc., 17:301-325, 1916.

[6] T. Takagi. A Simple Example of a Continuous Function Without Derivative. Proceedings of the Physico-Mathematical Society of Japan, 1(2):176-177, 1903.

[7] Masayoshi Hata. Singularities of the Weierstrass Type Functions. Journal d'Analyse Mathématique, 51, 1988.

[8] T-Y. Hu and K-S. Lau. Fractal Dimensions and Singularities of the Weierstrass Type Functions. Trans. of the American Mathematical Society, 335(2):649-665, 1993.

[9] R.D. Mauldin and S.C. Williams. On the Hausdorff Dimension of Some Graphs. Trans. of the American Mathematical Society, 298(2):793-803, 1986.

[10] S. Jaffard. Construction de Fonctions Multifractales ayant un Spectre de Singularités Prescrit. C.R. Acad. Sci. Paris, pages 19-24, 1992. T. 315, Serie I.

[11] W. Doeblin and R. Fortet. Sur des Chaînes à Liaisons Complètes. Bull. Soc. Math. de France, 65:132-148, 1937. 
[12] L. Dubins and F. Freedman. Invariant Probabilities for Certain Markov Process. Ann. Math. Statist., 37(1966):837-848.

[13] J. Hutchinson. Fractals and Self-Similarity. Indiana University Journal of Mathematics, 30:713-747, 1981.

[14] M. Barnsley. Fractal Functions and Interpolation. Constructive Approximation, 1985.

[15] E.R. Vrcsay. Iterated Function Systems: Theory, Applications and the Inverse Problem. Fractal Geometry and Analysis, pages 405-468, 1991.

[16] D.P. Hardin. Hyperbolic Iterated Function Systems and Applications. PhD thesis, Georgia Institute of Technology, 1985.

[17] Tim Bedford. Hölder Exponents and Box Dimension for Self-Affine Fractal Functions. Constructive Approximation, 5:33-48, 1989.

[18] S. Jaffard. Multifractal Formalism for Functions, Part 1 and Part 2. SIAM Journal of Mathematical Analysis. to appear.

[19] Jacques Lévy Véhel, Khalid Daoudi, and Evelyne Lutton. Fractal Modeling of Speech Signals. Fractals, 2(6), 1994.

[20] Khalid Daoudi and Jacques Lévy Véhel. Speech Modeling Based on Local Regularity Analysis. In Proceedings of the IASTED/IEEE International Conference on Signal and Image Processing, Las Vegas, USA, 20-23 November 1995.

[21] C.A. Rogers. Hausdorff Measures. Cambridge University Press, 1970.

[22] K. J. Falconer. Fractal Geometry : Mathematical Foundation and Applications. John Wiley \& Sons, 1990.

[23] Claude Tricot. Mesures et Dimensions. PhD thesis, Univ. Paris 11, 1983.

[24] Y. Meyer. Ondelettes. Hermann, 1990.

[25] S. Jaffard. Functions with Prescribed Hölder Exponent. Applied and Computational Harmonic Analysis, 2(4):400-401, October 1995.

[26] Claude Tricot. Courbes et Dimension Fractale. Springer-Verlag, 1993.

[27] Michael F. Barnsley. Fractals Everywhere. AK Peters, 1993.

[28] Leif M. Andersson. Recursive Construction of Fractals. Annales Academiae Scientiarum Fennicae, 1992.

[29] A. Arneodo, J.-F. Muzy, and E. Bacry. Multifractal Formalism for Fractal Signals. Technical report, Paul-Pascal Research Center, France, 1992. 